\title{
The linear spin-up of a stratified, rotating fluid in a square cylinder
}

\author{
M. R. FOSTER AND R. J. MUNR O $\mathrm{R}^{2}$ \\ ${ }^{1}$ Department of Mathematical Sciences, Rensselaer Polytechnic Institute, Troy, NY, 12180 \\ USA \\ ${ }^{2}$ Faculty of Engineering, University of Nottingham, Nottingham, NG7 2RD, UK
}

(Received ?; revised ?; accepted ?. - To be entered by editorial office)

Here we present experimental and theoretical results for how a stratified fluid, initially rotating as a solid body with constant angular velocity, $\Omega$, within a closed cylinder of square cross-section, is spun-up when subject to a small, impulsive increase, $\Delta \Omega$, in the cylinder's rotation rate. The fluid's adjustment to the new state of solid rotation can be characterised by (1) an inviscid, horizontal starting flow which conserves the vorticity of the initial condition, (2) the eruption of Ekman layer fluid from the perimeter region of the cylinder's base and lid, (3) horizontal-velocity Rayleigh layers that grow into the interior from container's sidewalls, and (4) the formation and decay of columnar vortices in the vertical corner regions. Asymptotic results describe the inviscid starting flow, and the subsequent interor spin-up that occurs due to the combined effects of Ekman suction through the base and lid Ekman layers, and the growth of the sidewall Rayleigh layers. Attention is focussed on the flow development over the spin-up time scale $T_{s}=E^{-\frac{1}{2}} \Omega^{-1}$, where $E$ is the Ekman number. (The spin-up process over the much longer diffusive time scale, $T_{d}=E^{-1} \Omega^{-1}$, is not considered here.) Experiments were performed using particle imaging velocimetry (PIV) to measure horizontal velocity components at fixed heights within the flow interior and at regular stages during the spin-up period. The velocity data obtained is shown to be in excellent agreement with the asymptotic theory.

\section{Introduction}

Consider a bounded, viscous, incompressible fluid rotating as a solid body with constant angular velocity $\Omega$. The process by which the fluid adjusts to a uniform change, $\Delta \Omega$, in the rotation rate of the flow boundaries is commonly known as spin-up $(\Delta \Omega / \Omega>0)$ or spin-down $(\Delta \Omega / \Omega<0)$. Given their significance to the dynamics controlling many geophysical and astrophysical flows, the spin-up and spin-down mechanisms have been studied for a variety of flow configurations and geometries, as well as for homogeneous, stratified and conducting fluids, resulting in a truly vast literature on the subject. Here, we choose not to attempt a summary of any but the most directly relevant work, since there have appeared two comprehensive reviews, first in Benton \& Clark (1974) and, more recently, in Duck \& Foster (2001).

Greenspan \& Howard (1963) were the first to provide a theoretical description of linear spin-up (i.e. with $0<\epsilon \ll 1$, where $\epsilon=\Delta \Omega / \Omega$ is the Rossby number) for the case of a homogeneous fluid confined within a closed, axially symmetric container. They showed that the increase in rotation rate of the container results in the formation of thin, quasisteady viscous Ekman layers at the horizontal base and lid, within which spun-up fluid is transported radially outward. To compensate for this radial mass flux, a weak axial flow (commonly referred to as Ekman suction) draws low angular momentum fluid from the inviscid interior into the upper and lower Ekman layers, which is in turn replaced by 
fluid from greater radii so that mass is conserved. Consequently, a secondary meridional circulation is established within the inviscid interior, drawing fluid radially inwards which, by conservation of angular momentum, acquires a greater rotational (or zonal) velocity. In addition, at the container corners, where the horizontal and vertical boundaries meet, the outward radial Ekman flux is deflected by the sidewall and discharged into, and transported by, the sidewall boundary layer (Stewartson 1957). The subsequent exchange of fluid from the Stewartson sidewall layer to the inviscid interior provides the inward radial mass flux needed to balance the axial flux into the Ekman layer.

Greenspan \& Howard (1963) showed the spin-up timescale for a homogeneous fluid to be $T_{s}=E^{-\frac{1}{2}} \Omega^{-1}$, where $E=\nu / \Omega L^{2}$ is the dimensionless Ekman number, $L$ a characteristic length scale and $\nu$ the kinematic viscosity of the fluid. That is, on this timescale the fluid is spun up by the action of the Ekman-layer driven secondary circulations described above. Eventually, the secondary circulations decay as the fluid approaches the spun-up state. Any small-scale residual oscillations that remain within the interior after this time are gradually damped by purely viscous effects over a diffusive timescale $T_{d}=E^{-1} \Omega^{-1}$. That is, during this final diffusive stage the vertical extent of the Ekman layer has grown to effectively fill the container. It is important to note that $E \ll 1$ in most practical situations, and so the diffusive timescale, $E^{-1} \Omega^{-1}$, is typically much longer than the corresponding spin-up timescale, $E^{-\frac{1}{2}} \Omega^{-1}$.

Walin (1969) and Sakurai (1969) considered the linear spin-up (and spin-down) of a linearly stratified rotating fluid within a closed circular cylinder, and showed that the fluid's vertical density gradient acts to inhibit the secondary Ekman-layer driven circulations described above. In particular, the presence of the fluid's density gradient means that the sidewall boundary layer is unable to transport the deflected radial Ekman flux (Duck \& Foster 2001), giving rise to a source-like eruption of spun-up Ekman-layer fluid from the corner region, into the interior. Despite this notable difference, Walin (1969) showed that the characteristic spin-up timescale for a stratified fluid is effectively the same as that for the homogenous case (i.e. $T_{s}=E^{-\frac{1}{2}} \Omega^{-1}$ ). However, unlike the homogenous case, the Ekman layer acts to spin-up the stratified fluid to a state of spatially non-uniform rotation, and the final uniform rotation rate is only attained on a much longer diffusive timescale of $\mathcal{O}\left(\sigma E^{-1} \Omega^{-1}\right)$, where $\sigma=\nu / \kappa$ is the Schmidt number with $\kappa$ denoting the diffusion coefficient of the stratifying agent.

The majority of spin-up studies (theoretical and experimental) have focussed primarily on axisymmetric configurations. However, van Heijst (1989) used experiments to analyze the non-linear spin-up from rest of a homogeneous free-surface fluid in a variety of nonaxisymmetric containers, including a semi-circular cylinder and an annular region with a radial barrier. This work was further extended by van Heijst et al. (1990) who considered the spin-up from rest of both homogeneous and linearly stratified fluids in a rectangular container (with length-to-width ratios of between 2 and 5), and by van de Konijnenberg \& van Heijst (1997) who analysed the spin-up from rest of a homogeneous fluid in a cylinder of square cross-section, with attention focussed on how free-surface deformation affects the evolution of the interior flow. Each of these studies found that the fluid's adjustment to a state of solid rotation could be characterised by three key stages, which are summarised here in relation to the pertinent case of a rectangular container (van Heijst et al. 1990). Firstly, at early times the boundary layers are still forming and so, relative to the inertial reference frame, the resulting starting flow is effectively inviscid and has zero absolute vorticity (when spun up from rest). In the co-rotating reference frame the starting flow takes the form of a single, central anticyclonic cell with closed streamlines that fill the flow domain. Furthermore, in the absence of Ekman suction, the starting 
flow is horizontal. During the second stage the boundary layers are established and the anticyclonic starting-vortex is now stretched by Ekman suction. Moreover, the boundary layers along each sidewall are observed to separate and detach near the corner regions, leading to the formation of corner cells consisting of vertical, cyclonic line vortices which extend over the tank depth. The cells downstream of the two long sides subsequently grow and interact with the central anticyclonic cell, giving rise to a three-dimensional, chaotic flow structure. During the final stage the background rotation leads to an eventual transition to two-dimensional motion, so that energy is transferred from small to large scales (i.e. the inverse energy cascade), resulting in the formation of an organised flow pattern consisting of an array of alternately cyclonic and anticyclonic cells, which gradually decay as the fluid is spun-up (and spun-down) by the Ekman layer below each cell.

This paper reports a combined theoretical and experimental investigation into the linear spin-up of a linearly stratified fluid contained in a (closed) square cylinder, which is initially in a state of solid rotation. In common with the flow described in the related study by van Heijst et al. (1990), the flow reported here is predominantly horizontal in nature. Hence, here, the experiments used two-dimensional particle imaging velocimetry (PIV) to measure the horizontal fluid velocity components in a horizontal plane section within the fluid's interior. Velocity measurements were taken throughout the flow development, from the initial starting flow following adjustment of the cylinder's rotation rate, and for a period of up to at least $2 \mathrm{hrs}$ (which typically corresponded to at least eight spin-up time scales, $T_{s}$ ). The velocity data were used for direct (quantitative) comparison with the theoretical results reported in this article. The PIV technique uses digital images taken of small particles suspended in the fluid interior, which act as a passive tracer. Hence, further insight into the qualitative features of the flow was obtained by recording streaklines (or time exposures) of the tracer particles, which provide estimates for the flow streamlines. The streakline data will be used to illustrate to the reader the key stages of the flow development.

There are a number of substantial differences between the dynamics of the flow reported here and that for an axisymmetric container, as reported in Walin (1969). A matter of great importance in all spin-up problems is the manner in which spun-up fluid in the Ekman layers affects spin-up in interior of the fluid. Let $\left(u^{*}, v^{*}\right)$ be dimensional horizontal velocity components - either Cartesian or polar. Then, the flow rate in the $d^{*}$-direction, per unit lateral distance, for example on the lower horizontal boundary, is

$$
\mathcal{Q}_{d^{*}}^{*}=-\frac{1}{2}\left(\frac{\nu}{\Omega}\right)^{\frac{1}{2}}\left(u^{*}+v^{*}-u_{b}^{*}-v_{b}^{*}\right),
$$

where $\left(u_{b}^{*}, v_{b}^{*}\right)$ are the boundary velocity components and $d^{*}$ is in the $x^{*}$-direction in Cartesian coordinates, or the radial $r^{*}$-direction in polar coordinates. (For the standard spin-up problem, where the adjustment in rotation rate is applied equally to all flow boundaries, we have $u_{b}^{*}=0, v_{b}^{*}=0$.) As Walin (1969) correctly points out, there is an Ekman-layer eruption in the axisymmetric case, over a region of width $\sqrt{\nu \Omega} t^{*}$, so that on the spin-up time scale, the fluid exchange between the Ekman layer and the invisicid interior occurs over an order-one region - because the radial Coriolis acceleration is zero at the vertical wall, and hence the azimuthal velocity component $v^{*}$ is zero there. If there is a differential rotation between the bottom surface and the sidewall, however, so that $u_{b}^{*} \neq 0$ and $v_{b}^{*} \neq 0$, then eruption persists over all time scales (Spence et al. 1992). The situation here is different: Because of the non-zero pressure-gradient parallel to the sidewall at $x^{*}=0$, for example, the tangential horizontal velocity component $v^{*}$ there does not vanish, and hence there is non-zero Ekman flux into the corner on the spin- 
up time scale, which must erupt into the interior. Accounting for these eruptions is an essential ingredient in getting the flow dynamics correct. So, the asymptotic theory for the spin-up problem considered in this article incorporates delta-function singularities in the corner regions.

Also, a prominent feature of the observed motion is the formation and decay of cyclonic corner eddies. These eddies form, as noted above, because the laminar boundary layer on the sidewalls has a finite-time eruption due to an adverse pressure gradient, and its cyclonic vorticity is shed into the corners. We show here that such eddy formation will occur at any Rossby number, no matter how small, at sufficiently long times. By contrast, in homogeneous spin-up in a rectangular tank, there is a critical Rossby number that scales with $E^{\frac{1}{2}}$, below which there is no eddy formation.

The structure of the paper is as follows: In $\S 2$, we describe the experiment apparatus, methodology and measurement techniques, and we discuss the observed flow features as they develop in time. In $\S 3$, we present the theoretical treatment of the linearized spinup problem in this geometry, including the boundary layers on the sidewalls. We also present here detailed comparisons of horizontal velocity profiles from the experiments and the theory. In $\S 4$, we turn briefly to the nonlinear sidewall boundary layers and their connection to eddy formation. Final discussions and concluding remarks are presented in $\S 5$.

\section{Experiment apparatus and observations}

\subsection{Apparatus and flow measurement}

Figure 1 shows a sketch of the basic apparatus and set-up. Each experiment was performed in a closed cylinder with a square cross-section of internal width $L=38.0 \mathrm{~cm}$ and height $H=51.0 \mathrm{~cm}$. The cylinder was mounted on a variable-speed turntable with its central vertical axis aligned with the rotation axis of the table. A standard double-reservoir system (Oster 1965) was used to fill the cylinder with a linearly stratified salt-water solution with buoyancy frequency $N=\left[g\left(\rho_{b}-\rho_{\ell}\right) / \rho_{\ell} H\right]^{\frac{1}{2}}$, where $\rho_{b}$ and $\rho_{\ell}$ denote the fluid densities at the base and lid of the cylinder, respectively. The turntable was then gradually brought, from rest, to the initial, steady rotation rate, $\Omega$, by applying small incremental increases in angular velocity over a time interval of 9-12 hrs. (In all experiments reported here the table was set to rotate in the clockwise direction.) The table and cylinder were then left to spin at the initial rotation rate $\Omega$ for a period of at least $16 \mathrm{hrs}$ $\left(\approx 0.4 E^{-1} \Omega^{-1}\right)$ to allow the fluid to attain a state of near solid-rotation. Any small-scale circulations still evident after this period were insignificant.

With this set-up procedure complete, each experiment was initiated (at time $t^{*}=0$ ) by applying a small, quasi-impulsive increase, $\Delta \Omega$, in the rotation rate of the table. The resulting relative motion of the fluid was observed and measured using particle imaging velocimetry (PIV), which is described in detail below. The important parameters for the experiments reported here are listed in table 1 , where note that attention was focussed on the case of small Rossby number $\epsilon=\Delta \Omega / \Omega$ (in the range 0.01-0.11) with the Burger number $S=(N / \Omega)^{2}$ of order one. Here, the Ekman number is defined as $E=\nu / \Omega L^{2}$ and in all cases was $\mathcal{O}\left(10^{-5}\right)$. For the range of salt concentrations used the kinematic viscosity $(\nu)$ and salt diffusivity $(\kappa)$ were constant, giving a constant Schmidt number $\sigma=\nu / \kappa \approx 670$ (Munro et al. 2010). Henceforth, we denote the aspect ratio of the cylinder's height and width by $h=H / L$, which here was fixed at $h=1.34$.

As the fluid adjusts to the increased rotation rate of the cylinder, the induced flow is predominantly horizontal. Hence, two-dimensional PIV was used to obtain measurements 


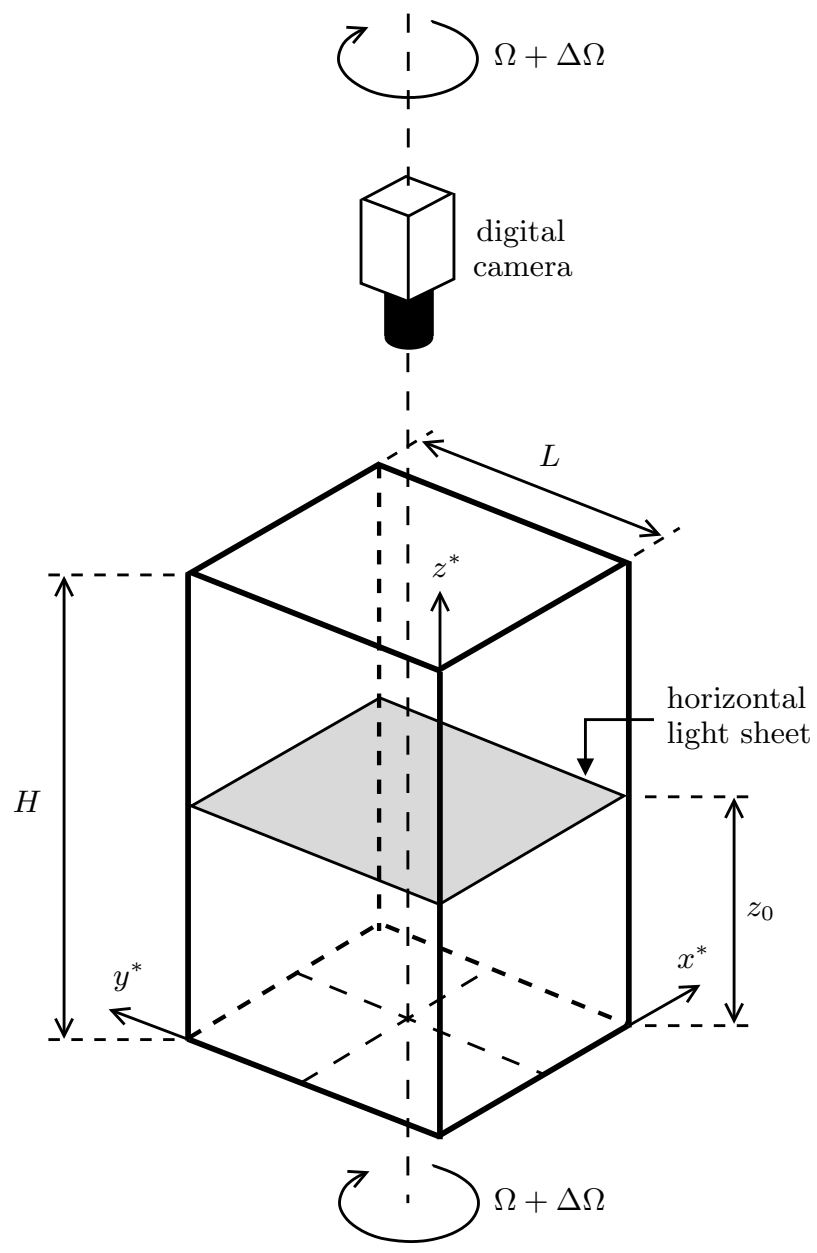

FiguRE 1. Sketch of the basic flow configuration and experiment set up. Also shown is the dimensional coordinate system, $\left(x^{*}, y^{*}, z^{*}\right)$, used to describe the flow.

of the fluid velocity field in a fixed horizontal plane within the tank interior during the spin-up period, and in the coordinate frame rotating with the cylinder. Small, reflective seeding particles (with mean density $1.026 \mathrm{~g} / \mathrm{cm}^{3}$ ) were added to the stratified solution during the filling process, and allowed to settle into suspension at their neutral-buoyancy level within the fluid interior, at a mean height $z_{0}$ above the cylinder base. Several values of $z_{0}$ were used (see the penultimate column of table 1 ), which was set by adjusting the reference densities $\rho_{b}$ and $\rho_{\ell}$. A slotted light box, containing two xenon arc lamps equipped with cylindrical optics, was mounted on the turntable and used to generate a thin light sheet directed through the container sidewall to illuminate the seeding particles within the horizontal plane at height $z_{0}$ (see figure 1). As the fluid was spun-up, the relative motion of the illuminated seeding particles was recorded using a co-rotating digital video camera (with $1280 \times 1024$ pixel resolution and sampling at $30 \mathrm{~Hz}$ ) attached to the turntable and positioned to view vertically down into the tank interior (see figure 1). The camera was controlled by a remotely operated laptop mounted on the turntable, which prevented the need for any physical contact with the table during the experiments. Image sequences were captured in bursts of between 30-90 sec and then transferred from 


$\begin{array}{cclcccccc}\text { Exp } & \Omega[\mathrm{rad} / \mathrm{s}] & \Delta \Omega[\mathrm{rad} / \mathrm{s}] & N[1 / \mathrm{s}] & \epsilon & S & E^{\frac{1}{2}} & z_{0} / H & T_{s}[\mathrm{~min}] \\ \mathrm{A} & 0.180 & 0.0193 & 0.924 & 0.107 & 26.4 & 0.00621 & 0.363 & 14.9 \\ \mathrm{~B} & 0.319 & 0.00906 & 0.924 & 0.0284 & 8.40 & 0.00466 & 0.363 & 11.2 \\ \mathrm{C} & 0.428 & 0.0149 & 0.934 & 0.0348 & 4.76 & 0.00403 & 0.386 & 9.68 \\ \mathrm{D} & 0.610 & 0.00674 & 0.934 & 0.0110 & 2.34 & 0.00338 & 0.386 & 8.11 \\ \mathrm{E} & 0.405 & 0.00821 & 0.797 & 0.0203 & 3.88 & 0.00414 & 0.588 & 9.95 \\ \mathrm{~F} & 0.524 & 0.00820 & 0.797 & 0.0157 & 2.31 & 0.00363 & 0.588 & 8.75 \\ \mathrm{G} & 0.374 & 0.00840 & 0.822 & 0.0225 & 4.84 & 0.00430 & 0.167 & 10.4 \\ \mathrm{H} & 0.494 & 0.0101 & 0.810 & 0.0204 & 2.69 & 0.00374 & 0.167 & 9.01\end{array}$

TABLE 1. The important experiment parameters, where $T_{s}=E^{-\frac{1}{2}} \Omega^{-1}$.

the camera to the laptop for storage. Each experiment ran for at least $2 \mathrm{hrs}$ (i.e. for $\Omega E^{\frac{1}{2}} t^{*} \approx 8$ to 15 ), with the image bursts captured every 2-3 mins during the initial stages and then every 10-30 mins during the later stages as the fluid approached the spun-up state. At the end of each experiment the velocity data were generated from the raw images using standard PIV software. To provide additional insight into the flow structure and development, the raw images were also used to visualise flow streamlines by recording the streaklines (or time exposures) of the seeding particles. In each case, the streaklines were generated from exposures recorded over a duration of $30 \mathrm{sec}$.

Figures 2 and 3 show typical samples of streakline images taken at various times during experiments $\mathrm{C}$ and $\mathrm{H}$ (see captions for details). Figure 4 shows a selection of velocity and vorticity data, also taken from experiment $\mathrm{C}$. Here, we have chosen to show experiments $\mathrm{C}$ and $\mathrm{H}$ as they illustrate the flow evolution near both the cylinder's mid height and the near-base region (at $z_{0}=0.386 H$ and $z_{0}=0.167 H$, respectively), and are representative of the behaviour observed in all experiments listed in table 1. For reference purposes we henceforth let $\left(x^{*}, y^{*}, z^{*}\right)$ denote the dimensional Cartesian coordinate system defined relative to the new rotating frame of the cylinder (as shown in figure 1), with the $z^{*}$-axis directed vertically upwards and the central rotation axis of the table and cylinder located along $\left(x^{*}, y^{*}, z^{*}\right)=\left(L / 2, L / 2, z^{*}\right)$. The base plane of the cylinder is at $z^{*}=0$. We now describe the key stages of the flow development.

\subsection{Observations}

As discussed previously in van Heijst et al. (1990), the increase in rotation rate of the cylinder results in a starting flow which, relative to the co-rotating reference frame, takes the form of a single, symmetric anticyclonic cell centred about the cylinder's rotation axis, with closed streamlines that fill the flow domain. The key features of the starting flow are shown figures 2(a), 3(a) and 4(a), where, recall, here the anticyclonic direction is anticlockwise. During the first one or two rotation periods the Ekman layers and sidewall boundary layers are still forming and so, as yet, have no significant influence on the interior flow. As a consequence, at early times the starting flow within the interior is effectively inviscid and, in the absence of Ekman suction, remains horizontal. Hence, relative to the co-rotating reference frame, the interior inviscid starting flow preserves the vorticity of the initial condition, which for the case of clockwise rotation considered here is given by $\boldsymbol{\omega}^{*}=\left(0,0, \varpi^{*}\right)=2 \Delta \Omega \hat{\mathbf{z}}$, where $\hat{\mathbf{z}}$ denotes the unit vector in the $z^{*}$-direction 


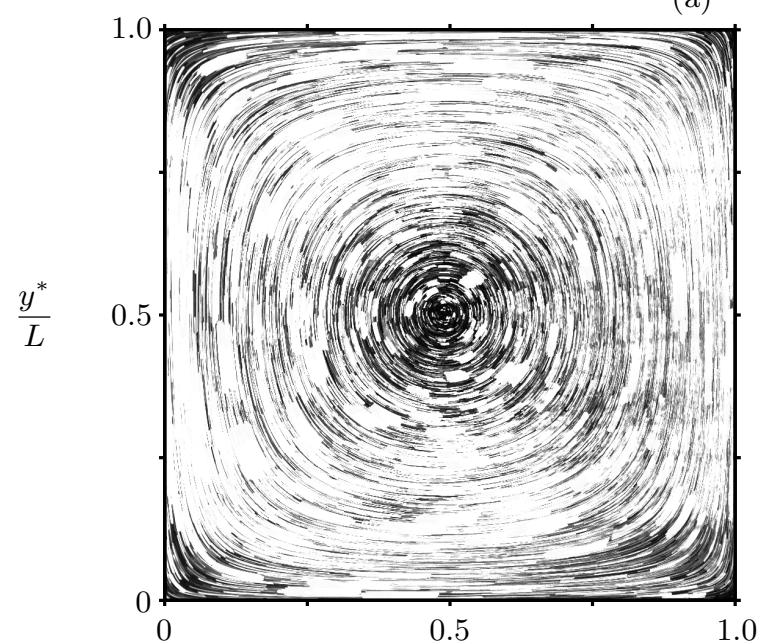

(c)

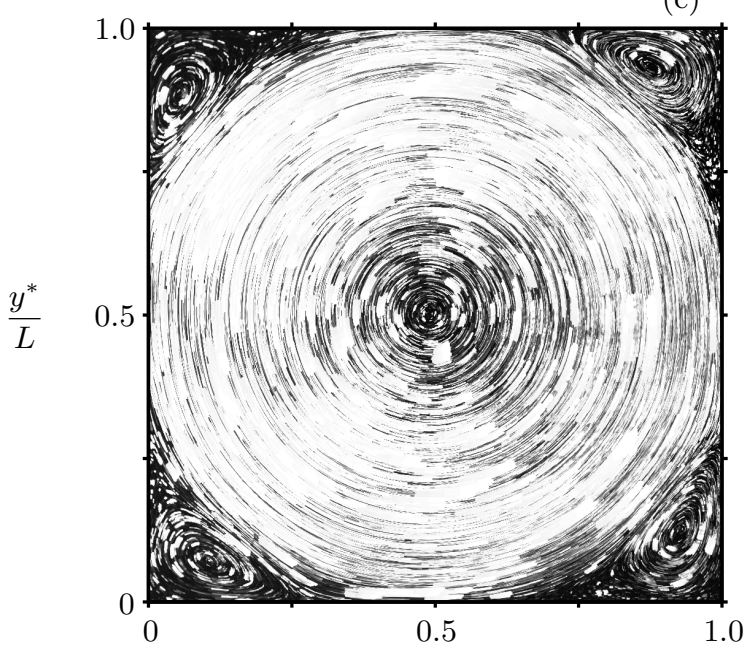

(e)

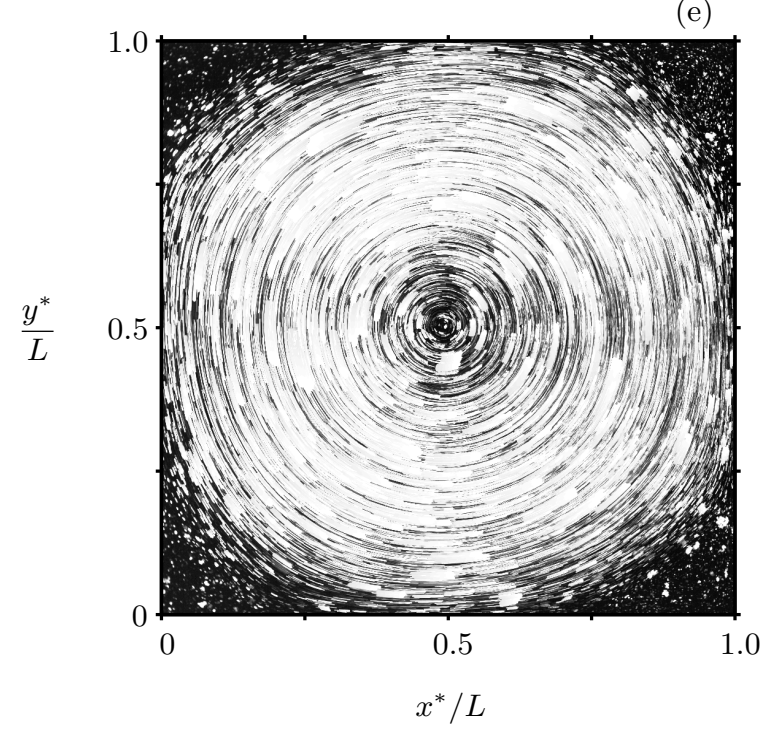

(b)

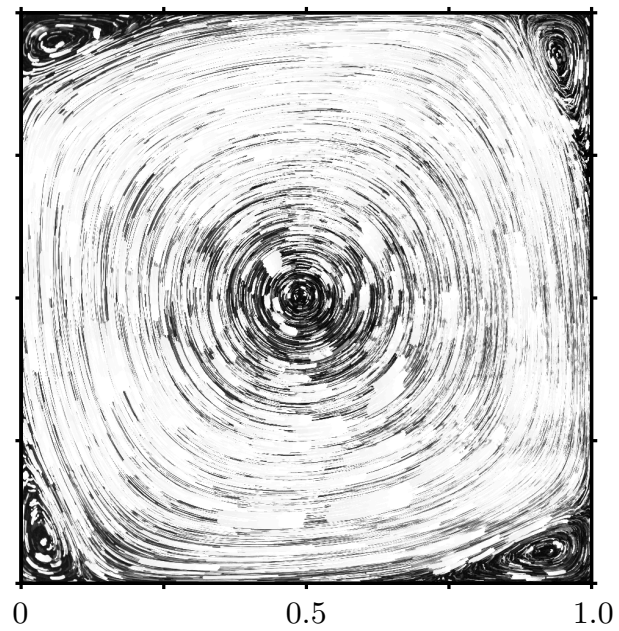

(d)

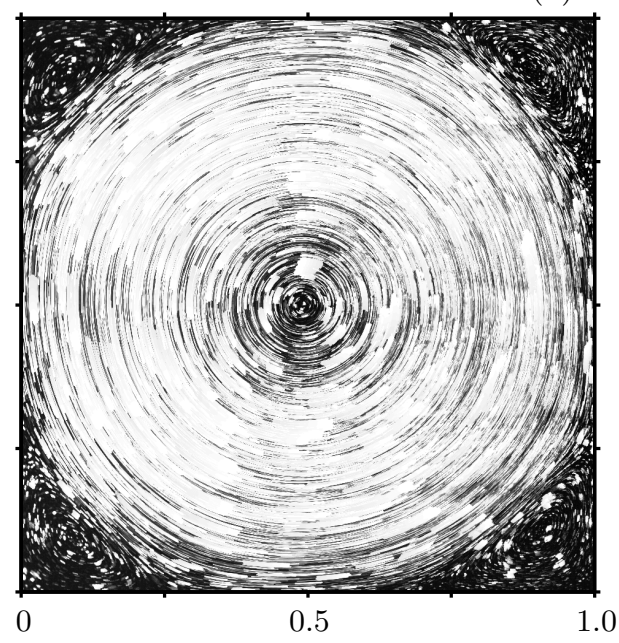

(f)

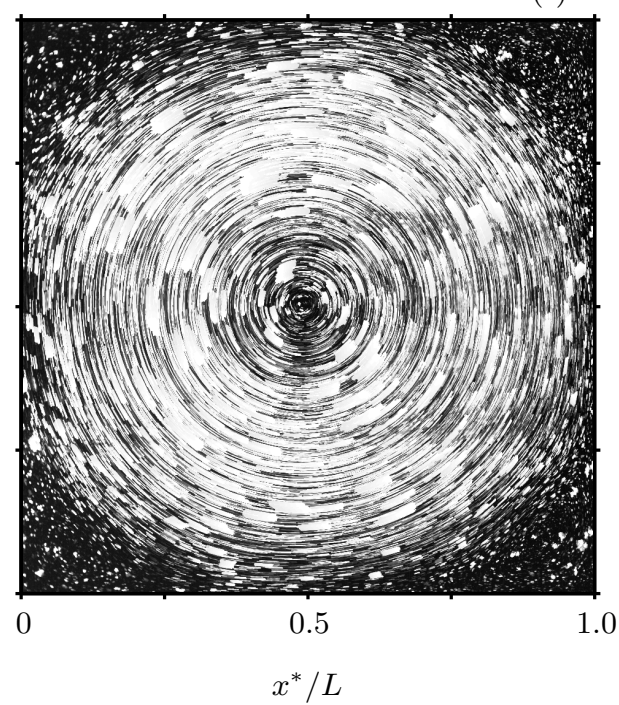

$x^{*} / L$

Figure 2. Experiment $\mathrm{C}\left(\Omega=0.428 \mathrm{rad} / \mathrm{s}, \epsilon=0.0348, S=4.76, z_{0}=0.386 H\right)$ : A selection of streakline images (obtained from $30 \mathrm{sec}$ exposures) taken at various times throughout the experiment: Images (a)-(f) correspond, respectively, to dimensionless times $\Omega t^{*}=8.6,43,120$, 370,650 and 1200 (or, $\Omega E^{\frac{1}{2}} t^{*}=0.035,0.17,0.47,1.5,2.6$ and 4.7 ). 
(a)

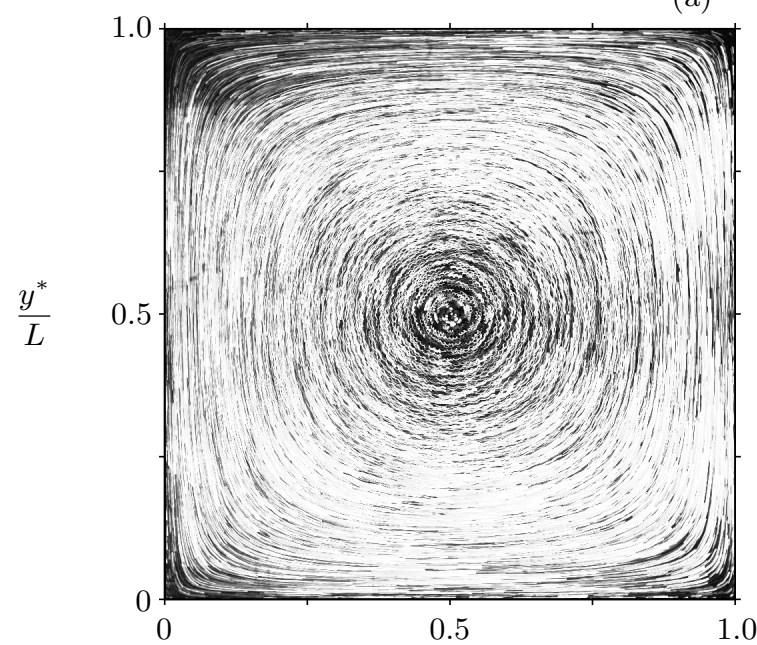

(c)

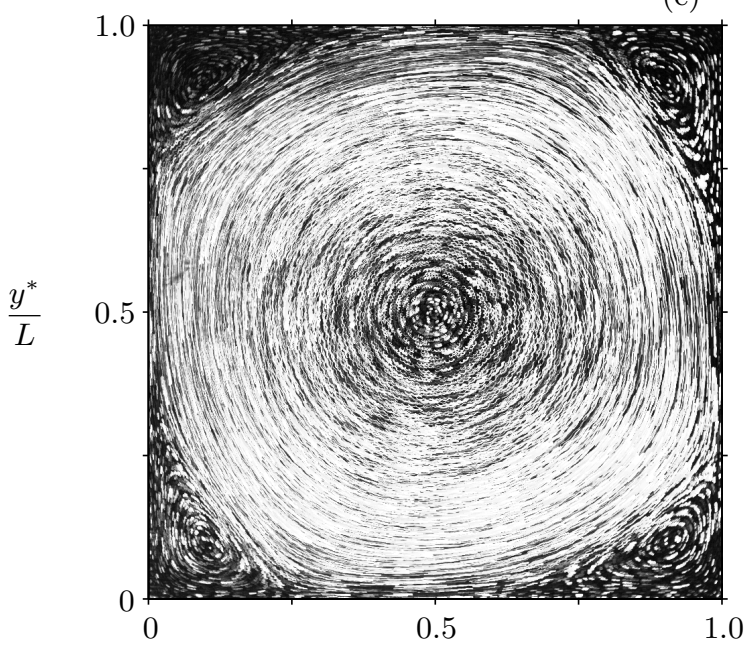

(e)

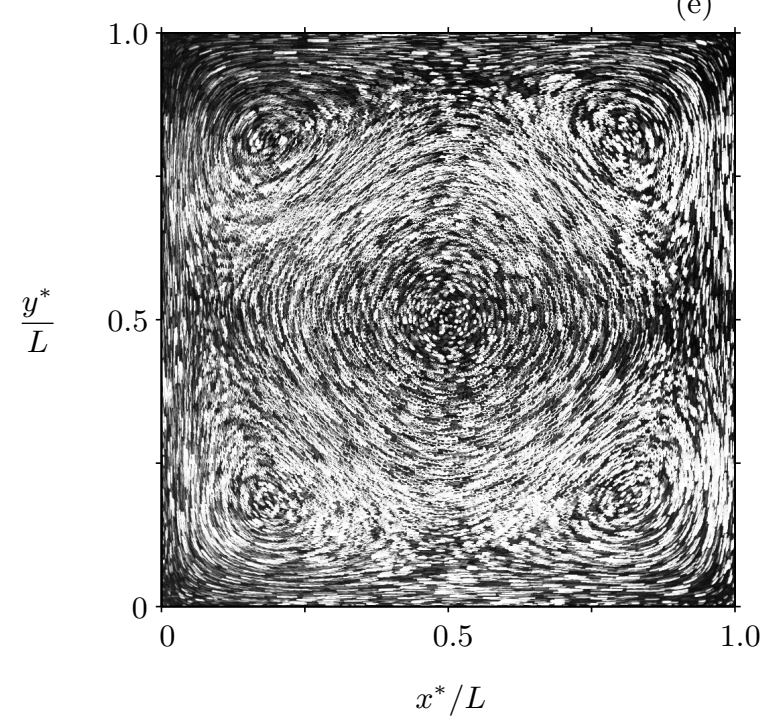

(b)

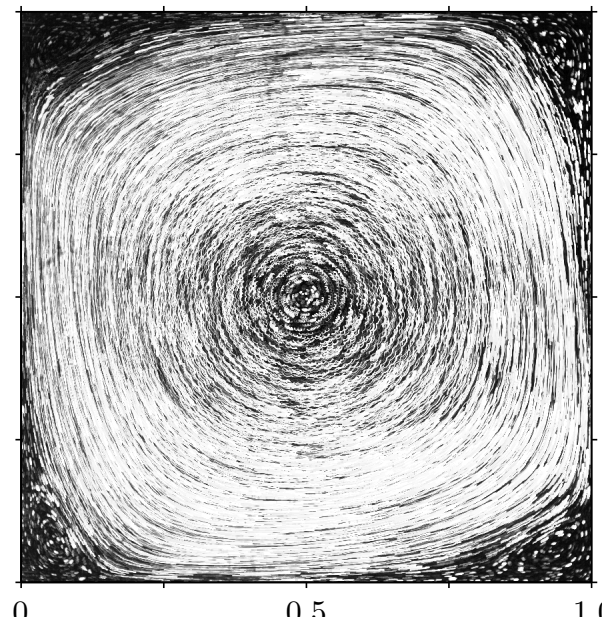

(d)

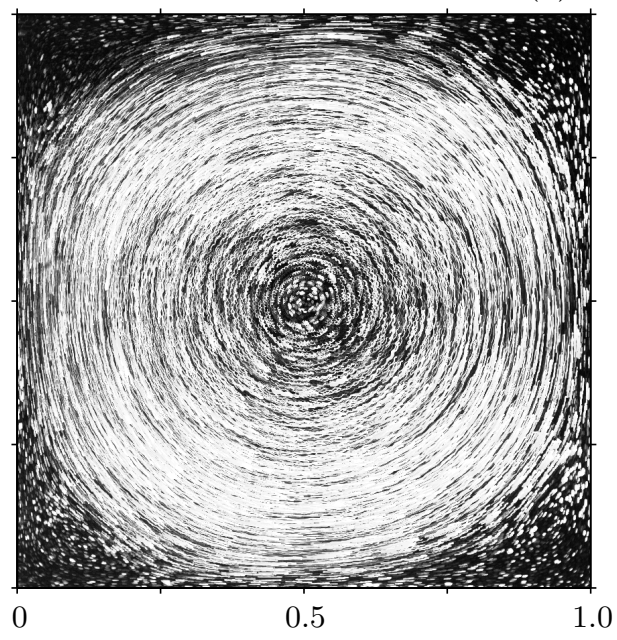

(f)

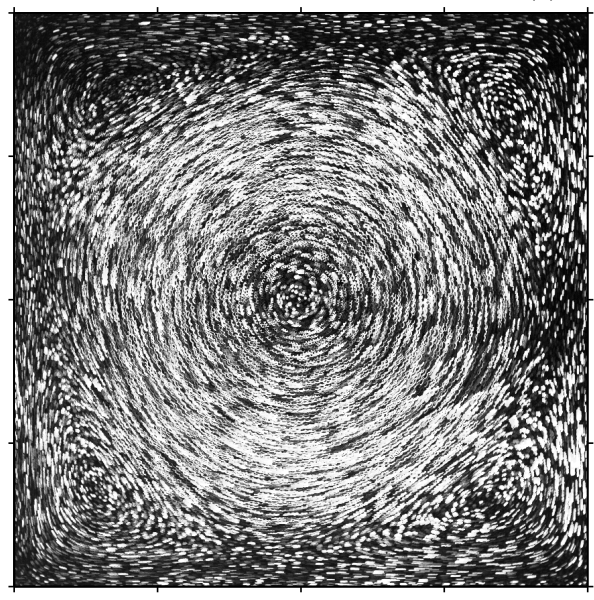

0.5

1.0

Figure 3. Experiment $\mathrm{H}\left(\Omega=0.494 \mathrm{rad} / \mathrm{s}, \epsilon=0.0204, S=2.69, z_{0}=0.167 H\right)$ : A selection of streakline images (obtained from $30 \mathrm{sec}$ exposures) taken at various times throughout the experiment: Images (a)-(f) correspond, respectively, to dimensionless times $\Omega t^{*}=8.4,82,160$, 300,590 and 900 (or, $\Omega E^{\frac{1}{2}} t^{*}=0.031,0.30,0.58,1.1,2.2$ and 3.4). 
(a)

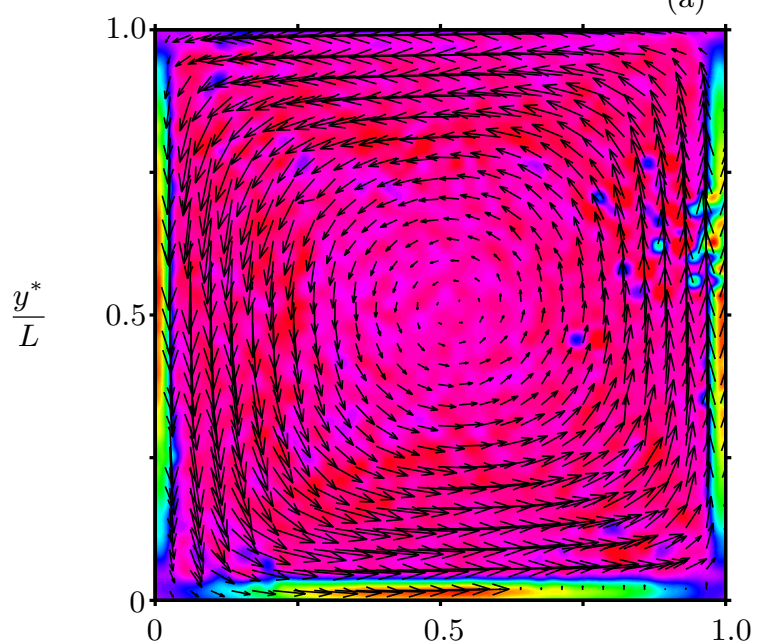

(c)

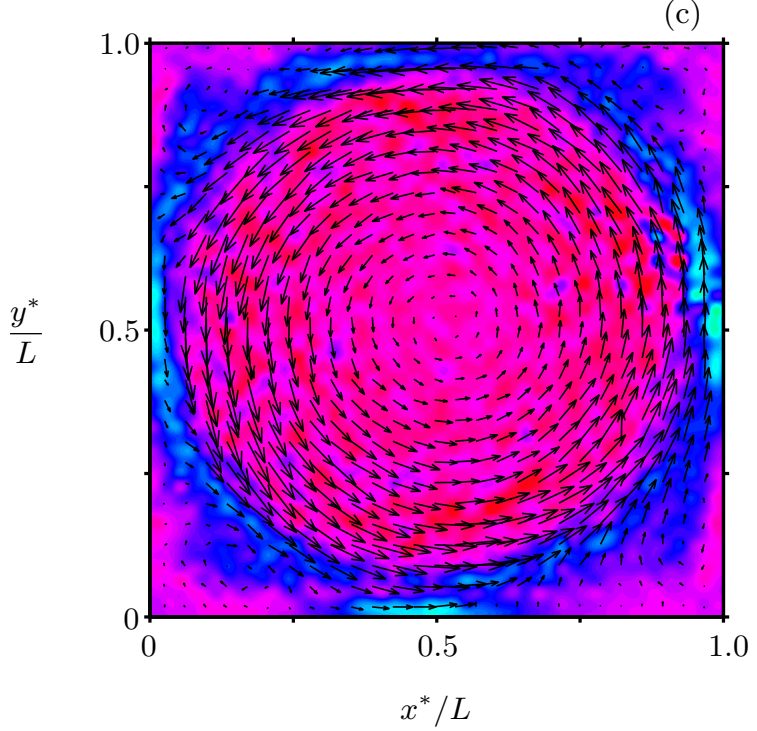

(b)

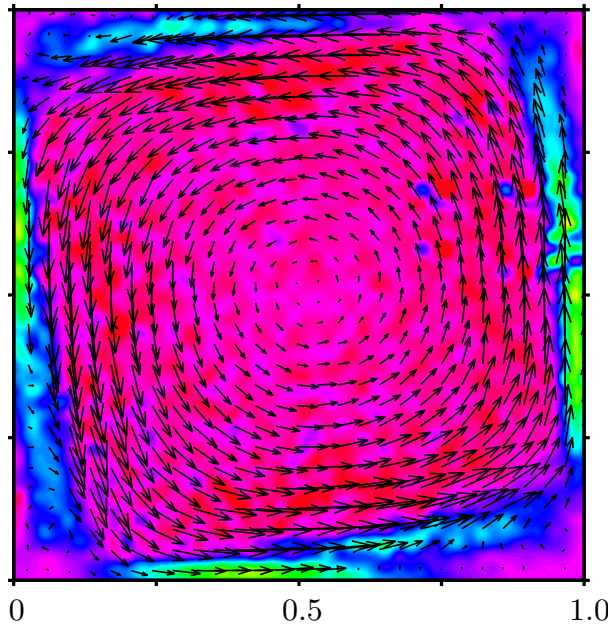

(d)

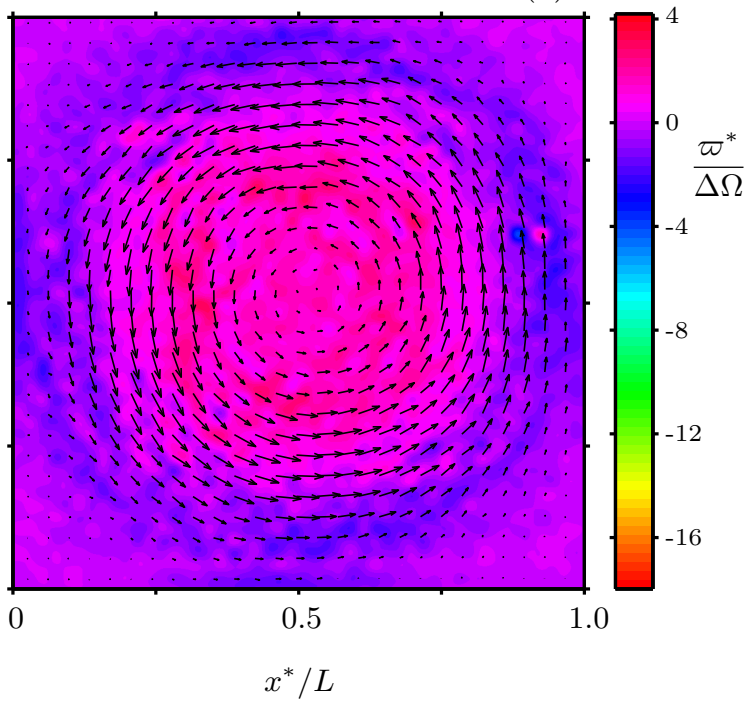

Figure 4. Experiment $\mathrm{C}\left(\Omega=0.428 \mathrm{rad} / \mathrm{s}, \epsilon=0.0348, S=4.76, z_{0}=0.386 \mathrm{H}\right)$ : A selection of PIV measurements of the horizontal-plane velocity components $\left(u^{*}, v^{*}\right)$ and the corresponding vertical vorticity $\varpi^{*}=v_{x^{*}}^{*}-u_{y^{*}}^{*}$, obtained at various times during the experiment: Plots (a)-(d) correspond, respectively, to dimensionless times $\Omega t^{*}=8.6,43,120$, and 650 (or, $\Omega E^{\frac{1}{2}} t^{*}=0.035$, $0.17,0.47$, and 2.6), and to the streakline images shown in figures $2(\mathrm{a}), 2(\mathrm{~b}), 2(\mathrm{c})$ and $2(\mathrm{e})$. The colour scale for the dimensionless vorticity $\varpi^{*} / \Delta \Omega$ is shown at the side of plot (d).

and $\varpi^{*}=v_{x^{*}}^{*}-u_{y^{*}}^{*}$ is the vertical vorticity component. Note that, in figure 4 the vorticity scale is shown in dimensionless form (i.e. $\varpi^{*} / \Delta \Omega$ ).

After several rotation periods the Ekman and sidewall boundary layers are now established, and for subsequent times the vorticity of the starting flow is stretched by Ekman suction. Within the first few rotation periods the sidewall boundary layers separate and eventually detach upstream of each corner region, ejecting cyclonic vorticity which is advected by the retrograde interior flow to accumulate within the sidewall corner regions. This leads to the formation of cyclonic line vorticies in each of these corner regions, which can be seen clearly in figures $2(\mathrm{~b})$ and $3(\mathrm{~b})$. As is the case with the initial starting flow, 

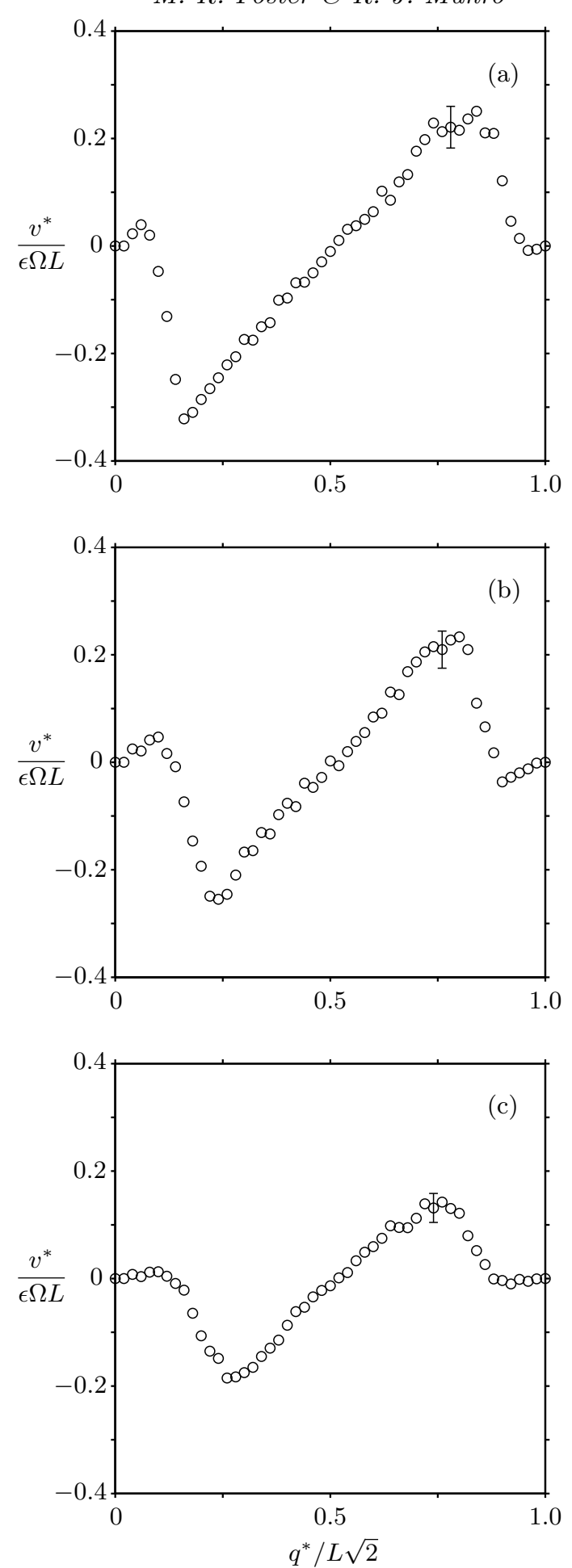

FIgURE 5. Experiment $\mathrm{C}\left(\Omega=0.428 \mathrm{rad} / \mathrm{s}, \epsilon=0.0348, S=4.76, z_{0}=0.386 H\right)$ : Measured values of the horizontal velocity component $v^{*}$ extracted across the diagonal $q^{*}=\left\{\left(x^{*}, y^{*}\right): y^{*}=x^{*}\right.$ for $\left.x^{*} \in[0, L]\right\}$. Plots (a)-(c) correspond, respectively to dimensionless times $\Omega t^{*}=43,120$ and 370 (or, $\Omega E^{\frac{1}{2}} t^{*}=0.17,0.47$ and 1.5 ). Here, $q^{*}$ has been made dimensionless by the diagonal length $L \sqrt{2}$, and $v^{*}$ by the velocity scale $\epsilon \Omega L$. The error bar shown in each plot is characteristic of the variability observed in each data set. 

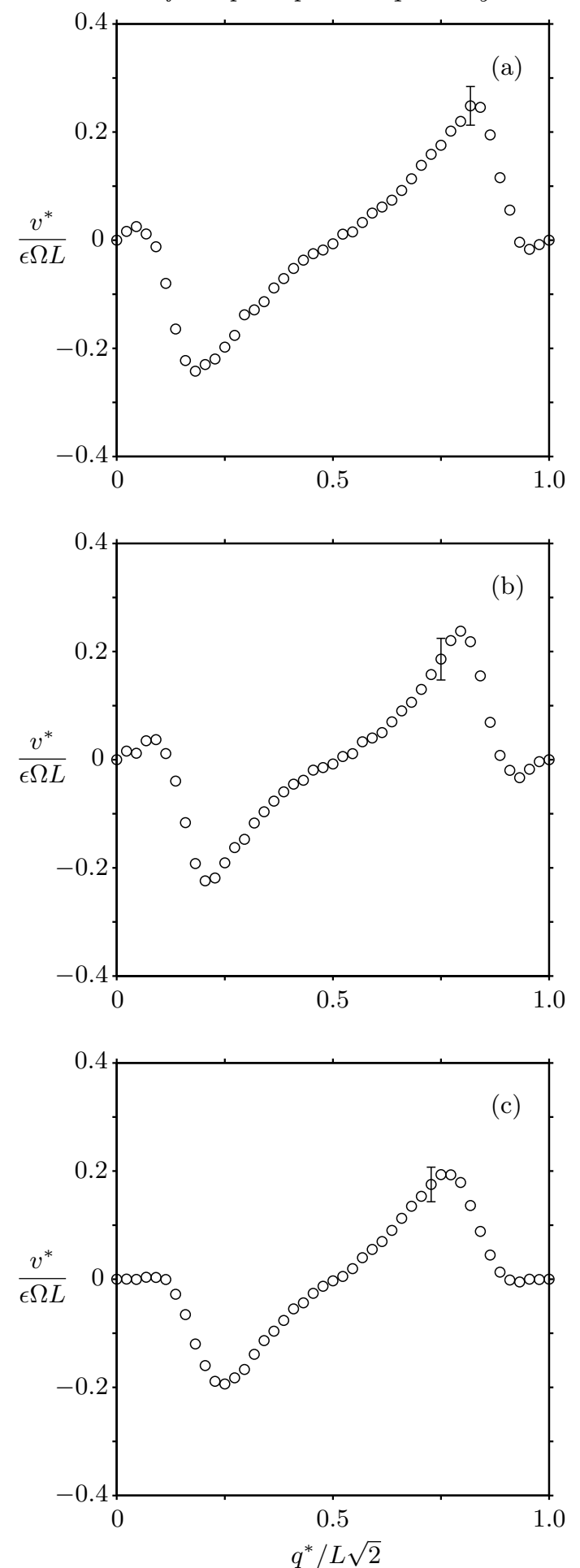

FIgURE 6. Experiment $\mathrm{H}\left(\Omega=0.494 \mathrm{rad} / \mathrm{s}, \epsilon=0.0204, S=2.69, z_{0}=0.167 \mathrm{H}\right)$ : Measured values of the horizontal velocity component $v^{*}$ extracted across the diagonal $q^{*}=\left\{\left(x^{*}, y^{*}\right): y^{*}=x^{*}\right.$ for $\left.x^{*} \in[0, L]\right\}$. Plots (a)-(c) correspond, respectively to dimensionless times $\Omega t^{*}=82,160$ and 300 (or, $\Omega E^{\frac{1}{2}} t^{*}=0.30,0.58$ and 1.1). Here, $q^{*}$ has been made dimensionless by the diagonal length $L \sqrt{2}$, and $v^{*}$ by the velocity scale $\epsilon \Omega L$. The error bar shown in each plot is characteristic of the variability observed in each data set. 

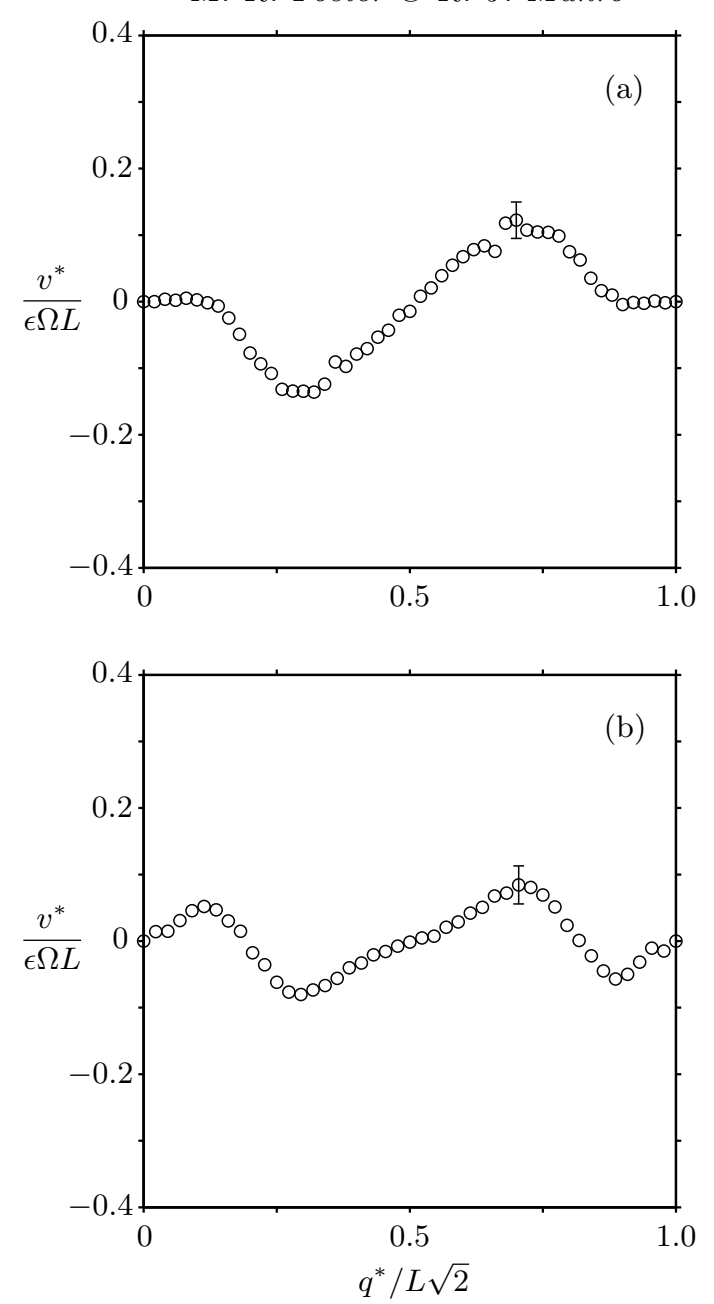

FIGURE 7. Measured values of the horizontal velocity component $v^{*}$ extracted across the diagonal $q^{*}=\left\{\left(x^{*}, y^{*}\right): y^{*}=x^{*}\right.$ for $\left.x^{*} \in[0, L]\right\}$. Plot (a) shows data from experiment $\mathrm{C}$ at time $\Omega E^{\frac{1}{2}} t^{*}=2.6$ (or, $\Omega t^{*}=650$, which corresponds the streakline data in figure 2e), and plot (b) shows data from experiment $\mathrm{H}$ at time $\Omega E^{\frac{1}{2}} t^{*}=2.2$ (or, $\Omega t^{*}=590$, which corresponds the streakline data in figure 3e). Here, $q^{*}$ has been made dimensionless by the diagonal length $L \sqrt{2}$, and $v^{*}$ by the velocity scale $\epsilon \Omega L$. The error bar shown in each plot is characteristic of the variability observed in each data set.

the vertical axis of these corner vorticies extends over the container depth. For the experiments reported here the presence of the corner cells was typically first observed after $\Omega t^{*} \approx 30$ (i.e. four or five rotation periods). As more cyclonic vorticity accumulates in these regions the four corner cells grow in cross-section, while remaining equal in size to one another, and gradually deform the outer perimeter of the central cell (figures 2c and $3 \mathrm{c})$. Further illustration of the corner-cell development is provided by figures 5 and 6 , which show measured values of the horizontal velocity component $v^{*}$ extracted along the diagonal $q^{*}=\left\{\left(x^{*}, y^{*}\right): y^{*}=x^{*}\right.$ for $\left.x^{*} \in[0, L]\right\}$, at three separate times (see captions for details). The velocity profiles shown in figure 5 , which are taken from experiment $\mathrm{C}$, correspond to the respective streakline images shown in figures 2(b), 2(c) and 2(d). Likewise, the profiles shown in figure 6 are from experiment $\mathrm{H}$ and correspond, respectively, 
to figures 3(b), 3(c) and 3(d). In both cases, the corner cells are clearly evident by the change in sign of $v^{*}$ within the corner regions, which also confirms that the vorticity of the corner cells is cyclonic compared to the anticyclonic vorticity of interior, central cell. Figures 5 and 6 also highlight that the measured peak flow speeds in the four corner cells were always significantly smaller (typically less than $20 \%$ for the experiments reported here) than the corresponding peak flow speeds observed within the interior, central cell. At the later times shown in figures $5(\mathrm{c})$ and $6(\mathrm{c})$, which corresponds to $\Omega E^{\frac{1}{2}} t^{*} \approx 1$, we see that the corner cells have reached an essentially fully spun-down state, while there remains notable motion within the central cell, which is still being spun-up.

Eventually, a quasi-steady state is reached, as shown in figures $2(\mathrm{~d})$ and $3(\mathrm{~d})$, where the flow consists of the deformed anticyclonic central cell, which due to the symmetry of the corner-cell formation is now essentially circular in cross-section, and the four spundown corner regions. At heights away from the cylinder's base (and lid), this basic flow structure persists for subsequent times, as the relative flow of the central cell is gradually spun-up by the combined action of Ekman suction through the base and lid Ekmanlayers and, as will be shown in $\S 3$, by the horizontal growth of the sidewall boundary layers into the interior region. Figures $2(\mathrm{~d}-\mathrm{f})$ show the gradual decay of the central cell at height $z_{0}=0.386 H$, over several spin-up timescales, and this is typical of the behaviour observed in experiments A to F, each of which correspond to heights about the cylinder's mid-plane.

A somewhat different behaviour is observed at heights close to the cylinder's base (and lid). This is best illustrated by the streakline data shown in figure 3 (from experiment $\mathrm{H}$, at height $\left.z_{0}=0.167 \mathrm{H}\right)$, and by comparing the two velocity profiles in figure 7 , which show measured values of the horizontal velocity component $v^{*}$, extracted along the diagonal $q^{*}$, taken from (a) experiment $\mathrm{C}\left(z_{0}=0.386 H\right)$ at time $\Omega E^{\frac{1}{2}} t^{*}=2.6$, and (b) experiment $\mathrm{H}\left(z_{0}=0.167 H\right)$ at time $\Omega E^{\frac{1}{2}} t^{*}=2.2$. Note that the data in figures $7(\mathrm{a})$ and $7(\mathrm{~b})$ correspond, respectively, to the streakline images shown in figures 2(e) and $3(\mathrm{e})$. As stated above, at time $\Omega E^{\frac{1}{2}} t^{*} \approx 1$ the flow shown in figure $3(\mathrm{~d})$ consists of the central anticyclonic cell with the four spun-down corner regions. However, at time $\Omega E^{\frac{1}{2}} t^{*} \approx 2$ (figures $3 \mathrm{e}$ and $7 \mathrm{~b}$ ) we see that equally sized cyclonic cells have reformed in each of the corner regions. In particular, note that the peak flow speeds within the corner cells are similar to those observed at earlier times (which is evident by comparing figures $6 \mathrm{~b}$ and $7 \mathrm{~b}$ ), but now are comparable to the peak flow speeds observed within the central anticyclonic cell at this time. We reiterate here that this phenomenon was only observed in experiments $\mathrm{G}$ and $\mathrm{H}\left(\right.$ at $\left.z_{0}=0.167 \mathrm{H}\right)$, and not at the mid-heights considered in experiments A to F. For example, the velocity profile in figure 7 (a) (experiment C, $z_{0}=0.386 H$ ) at time $\Omega E^{\frac{1}{2}} t^{*}=2.6$ shows that once the corner cells are spun-down at this height, they subsequently remain spun-down. (See, also, the streakline image for experiment $\mathrm{C}$ in figure $2 \mathrm{f}$.)

The reasons for the corner-cell reformation in the region close to cylinder's base (and lid) was not clear from the experiment observations. However, it is likely they are caused by ejected fluid, not from the sidewall boundary layers, but from the eruption of Ekmanlayer fluid from the perimeter-region of the cylinder's base (and lid). The erupted fluid is advected by the retrograde central cell and accumulates in the sidewall corner-regions adjacent to the base (or lid). After several spin-up timescales (i.e. $\Omega E^{\frac{1}{2}} t^{*} \approx 2$ ) enough Ekman-layer fluid has erupted and entered these regions for the corner cells to have grown sufficiently in the vertical direction to be observed at height $z_{0}=0.167 \mathrm{H}$, as shown in figure 3(f). The "reformed" corner cells were observed to gradually decay over several spin-up timescales, so that after $\Omega E^{\frac{1}{2}} t^{*} \approx 3$ or 4 the flow in the region above 
the cylinder's base again consists of the central anticyclonic cell and the four spun-down corner regions, as shown in figure $3(\mathrm{f})$. Note that, at this time, the central cell is much weakened and approaching the spun-up state. Furthermore, comparing figures $2(\mathrm{f})$ and $3(\mathrm{f})$ shows that the fluid in the near-base region approaches the spun-up state more rapidly than the fluid around the cylinder's mid height, a feature in common with the case of axisymmetric spin-up of a stratified fluid (Walin 1969).

Finally, it is worth highlighting that the behaviour described here is notably different from that observed in the case of spin-up from rest in a rectangular container (van Heijst et al. 1990), where during the separation stage the corner cells form in an asymmetric way, with larger cells developing downstream of the long sides and small cells downstream of the short sides. As the larger corner cells grow they deform and eventually interact with the central anticyclonic cell, which leads to the formation a quasi-steady array of alternating cyclonic and anticyclonic cells which fill the container interior (the number of which depends on the aspect ratio of the container).

\section{Linear theory}

\subsection{Overview}

There are parametric restrictions in the theoretical analysis presented in this section. Unlike the situation for homogeneous spin-up problems, these restrictions are related to certain characteristic phenomena as they occur in time. In particular, there is the usual "spin-up time scale", $T_{s}=E^{-\frac{1}{2}} \Omega^{-1}$, and as is well known in stratified spin-up, the final processes occur over a diffusive scale, $T_{d}=E^{-1} \Omega^{-1}$. However, as discussed in $\S 2.2$, separation of the sidewall boundary layers leads to the formation of corner eddies, and that turns out to occur on a time scale $T_{f}=\epsilon^{-1} \Omega^{-1}$. So, here, we require

$$
T_{s} \ll T_{f} \ll T_{d},
$$

which then leads immediately to

$$
\epsilon \ll E^{\frac{1}{2}} \ll 1,
$$

which will be assumed to hold throughout the theoretical analysis presented in this section. Referring to table 1 shows that $E^{\frac{1}{2}} / \epsilon \approx 0.06$ to 0.31 for the experiments reported here. Despite this contradiction with the condition (3.2), we will show that the theoretical analysis based on (3.2) still shows good quantitative agreement with the experimental data.

\subsection{Problem formulation}

The flow configuration has been described in $\S 2$ and is shown schematically in figure 1. In addition, the stratified fluid will henceforth be assumed to be incompressible and Boussinesq. If the potential of the centrifugal force is neglected, the dimensional density $\left(\bar{\rho}^{*}\right)$ and pressure $\left(\bar{p}^{*}\right)$ can be written relative to the rotating coordinate system as

$$
\begin{gathered}
\bar{\rho}^{*}\left(\mathbf{x}^{*}, t^{*}\right)=\rho_{\ell}+\rho_{s}^{*}\left(z^{*}\right)+\rho^{*}\left(\mathbf{x}^{*}, t^{*}\right), \\
\bar{p}^{*}\left(\mathbf{x}^{*}, t^{*}\right)=p_{s}^{*}\left(z^{*}\right)+p^{*}\left(\mathbf{x}^{*}, t^{*}\right),
\end{gathered}
$$

where $\rho_{s}^{*}$ and $p_{s}^{*}$ denote the contributions associated with the background stratification, $\rho_{\ell}$ is a constant reference density (taken here to be the initial density at the container lid), and $\rho^{*}$ and $p^{*}$ are the perturbations arising due to the increase in rotation rate of the cylinder. It is assumed throughout that $\rho^{*} \ll \rho_{s}^{*}, \rho_{\ell}$. If the following dimensionless 


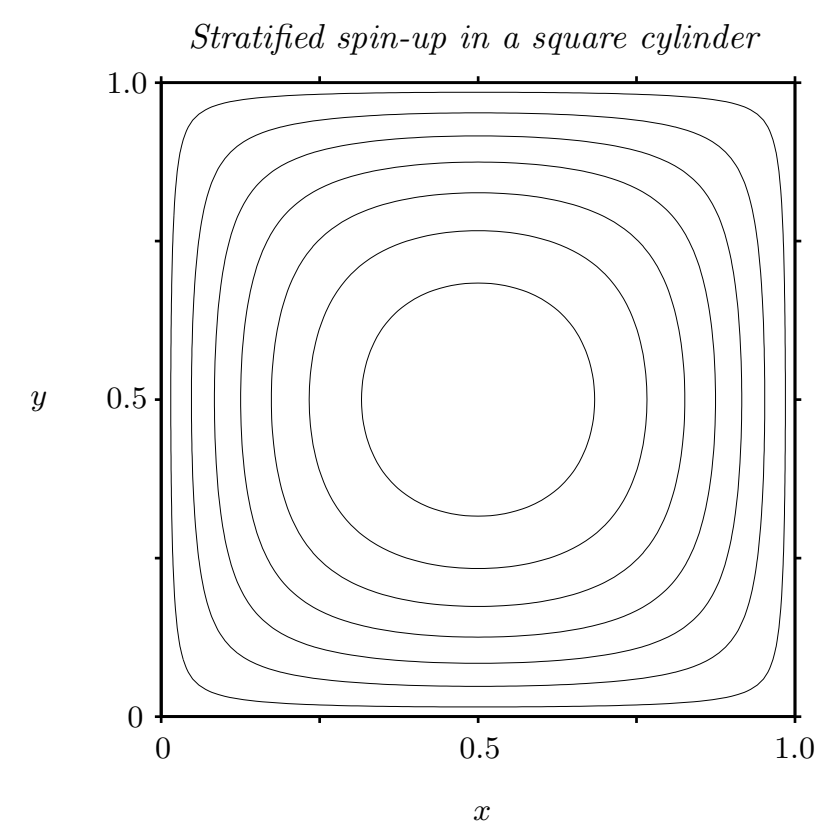

FiguRE 8. A sample of contours for the streamfunction $p(x, y)$ defined in equation (3.15).

variables

$$
\mathbf{u}=\frac{\mathbf{u}^{*}}{\epsilon \Omega L}, \mathbf{x}=\frac{\mathbf{x}^{*}}{L}, t=\Omega t^{*}, \rho=\frac{g \rho^{*}}{\epsilon \rho_{\ell} \Omega^{2} L}, p=\frac{p^{*}}{\epsilon \rho_{\ell} \Omega^{2} L^{2}},
$$

are introduced, where $\mathbf{u}(\mathbf{x}, t)$ is the velocity vector with components $(u, v, w)$, the timedependent governing equations may be written in dimensionless form as

$$
\begin{gathered}
\nabla \cdot \mathbf{u}=0 \\
\mathbf{u}_{t}+\epsilon(\mathbf{u} \cdot \nabla) \mathbf{u}+2(\hat{\mathbf{z}} \times \mathbf{u})+\rho \hat{\mathbf{z}}+\nabla p=E \nabla^{2} \mathbf{u}, \\
\rho_{t}+\epsilon(\mathbf{u} \cdot \nabla) \rho-S w=(E / \sigma) \nabla^{2} \rho,
\end{gathered}
$$

where the dimensionless Rossby $(\epsilon)$, Ekman $(E)$, Burger $(S)$ and Schmidt $(\sigma)$ numbers are, as noted above, defined as

$$
\epsilon=\frac{\Delta \Omega}{\Omega}, E=\frac{\nu}{\Omega L^{2}}, S=\left(\frac{N}{\Omega}\right)^{2}, \sigma=\frac{\nu}{\kappa} .
$$

The buoyancy frequency $N$ is assumed to remain constant throughout. Also, recall that the aspect ratio of the cylinder's height and width has been denoted by $h=H / L$, which for the experiments reported here is fixed at $h=1.34$. The corresponding initial and boundary conditions can be written (in dimensionless form) as

$$
\begin{gathered}
\mathbf{u}=\hat{\mathbf{z}} \times\left(\mathbf{x}-\mathbf{x}_{0}\right)=\left(\frac{1}{2}-y, x-\frac{1}{2}, 0\right), \rho=0 \text { for } t=0, \mathbf{x} \in \mathcal{D}, \\
\mathbf{u}=0, \frac{\partial \rho}{\partial n}=0 \text { for } \mathbf{x} \in \partial \mathcal{D}, t>0,
\end{gathered}
$$

where $\mathbf{x}_{0}$ denotes the position vector of the cylinder-base centre, $\mathcal{D}=\{(x, y, z): x, y \in$ $[0,1], z \in[0, h]\}$ is the flow domain, $\partial \mathcal{D}$ the domain boundary, and $n$ is used to represent the normal direction at each boundary. 

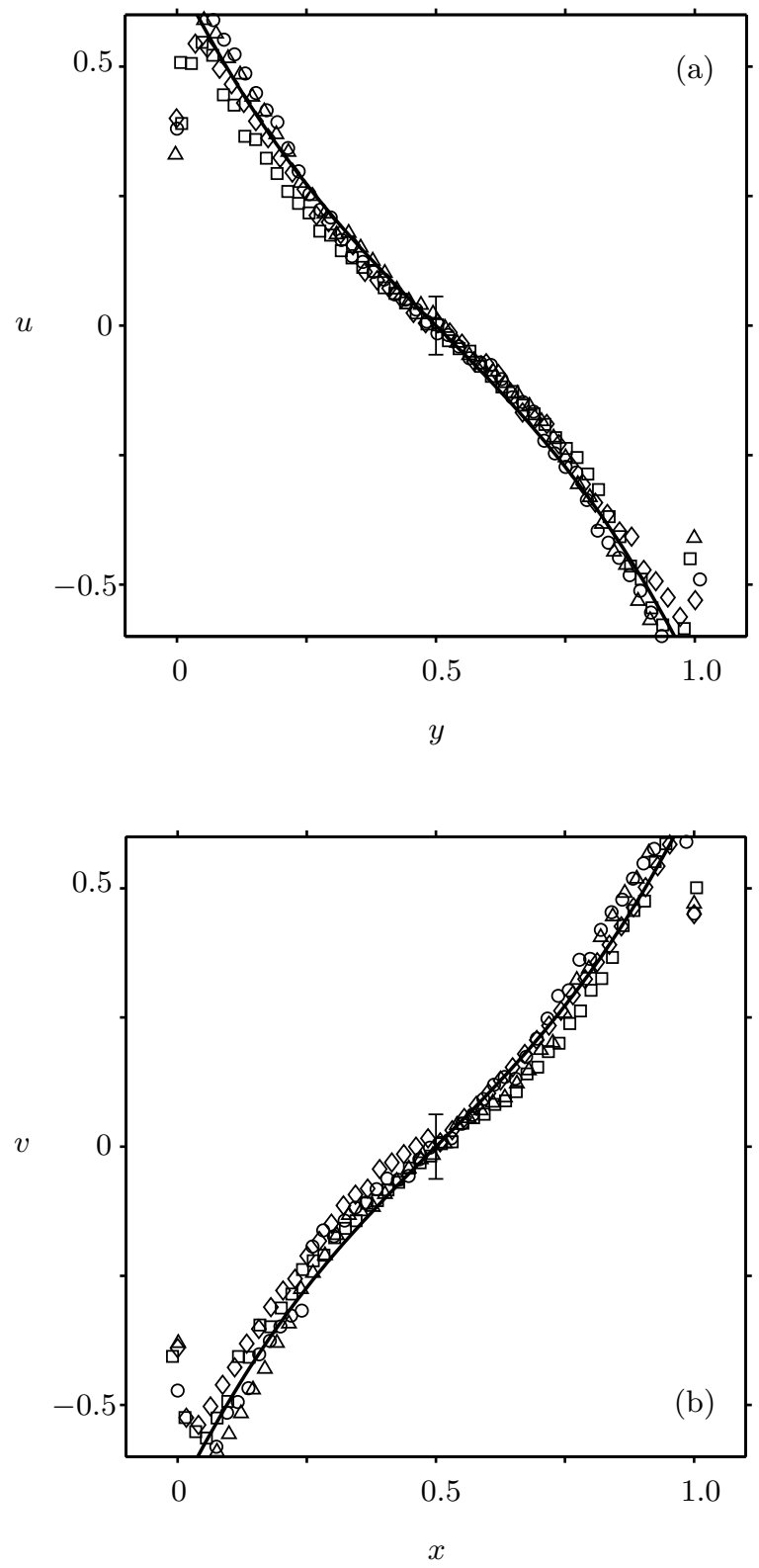

FiguRE 9 . The solid lines show the theoretical solution for the starting-flow velocity components $u(x, y)$ and $v(x, y)$, defined by equations (3.15) and (3.17) (with $m, n=150$ ), compared with corresponding data obtained from experiments A $(\square)$, C (o), E $(\triangle)$ and $\mathrm{G}(\diamond)$ : (a) shows $u(y)$ (along $x=1 / 2$ ) plotted against $y$, and (b) shows $v(x)$ (along $y=1 / 2$ ) plotted against $x$. The experimental data were obtained at dimensionless times $t=\Omega t^{*}=1.80(\operatorname{Exp} \mathrm{A}), 2.14(\operatorname{Exp} \mathrm{C})$, $1.21(\operatorname{Exp} \mathrm{E})$ and $1.12(\operatorname{Exp} \mathrm{G})$. The error bar in each plot is characteristic of the variability observed in all of the data shown.

\subsection{The inviscid interior flow}

If we now take $E, \epsilon$ to be small, and ignore regions of large gradient, then the equations (3.5) take the inviscid form

$$
\begin{gathered}
\nabla \cdot \mathbf{u}=0 \\
\mathbf{u}_{t}+2(\hat{\mathbf{z}} \times \mathbf{u})+\rho \hat{\mathbf{z}}+\nabla p=0, \\
\rho_{t}-S w=0 .
\end{gathered}
$$


A formal asymptotic expansion may be used, and these equations are valid for terms up to $\mathcal{O}(E)$. Taking the curl and the divergence of $(3.8 b)$, one obtains equations involving pressure, vertical vorticity and vertical velocity. Then, the $z$-component of $(3.8 b)$ may be used to eliminate the vertical velocity, and the resulting vorticity equation and Poisson equation for the pressure may be combined to give

$$
\frac{\partial^{3}}{\partial t^{3}}\left(\nabla^{2} p\right)+\frac{\partial}{\partial t}\left(S \nabla_{1}^{2} p+4 p_{z z}\right)=0
$$

where in the second term $\nabla_{1}^{2} \equiv \partial^{2} / \partial x^{2}+\partial^{2} / \partial y^{2}$ is the horizontal Laplacian. The velocity components are related to the pressure, in general, by

$$
u_{t t}+4 u=-p_{x t}-2 p_{y}, \quad v_{t t}+4 v=-p_{y t}+2 p_{x}, \quad w_{t t}+S w=-p_{z t} .
$$

The boundary condition at each horizontal wall is no-penetration, so long as the time scale is shorter than $E^{-\frac{1}{2}}$. The last of the above equations then gives

$$
p_{z t}=0 \text { at } z=0, h, \text { for } t=o\left(E^{-\frac{1}{2}}\right) .
$$

Equation (3.9) itself is valid for all times short of the diffusive time scale.

\subsection{The starting flow: $t \ll E^{-\frac{1}{2}}$}

Suppose that we wish to investigate the flow at early times, shortly after the container is spun up to its new angular velocity. Because of the lower and upper wall boundary conditions, (3.11), the solution for all $t$ smaller than the spin-up time scale is $z$-independent. Therefore, since, from the initial conditions both $\nabla^{2} p_{t}$ and $\nabla^{2} p_{t t}$ are zero, and the initial value of $\nabla_{1}^{2} p$ is 4 , equation (3.9) reduces simply to

$$
\nabla_{1}^{2} p=4
$$

subject to the boundary conditions deduced from (3.10). Note that, on the $x=0$ boundary, for example, for $t \ll 1$,

$$
p_{x}=0 .
$$

Such Neumann conditions all around lead to a double Fourier cosine series solution of the Poisson equation (3.12). It turns out all terms except the average term are zero-that is, no spin-up has yet begun at times of this order. For $t=\mathcal{O}(1)$, there clearly are mixed boundary conditions, but for $1 \ll t \ll E^{-1 / 2}$, the boundary condition becomes $p_{y}=0$, and therefore $p=0$ on $x=0$. Such a Dirichlet condition is then the correct one all around the boundary, and hence, for these times,

$$
p=0 \text { on } x=0,1,0 \leqslant y \leqslant 1 \text { and on } y=0,1,0 \leqslant x \leqslant 1 .
$$

This formulation is equivalent to that found in van Heijst (1989) and van Heijst et al. (1990) for other asymmetric geometries. The solution to (3.12) and (3.14) is

$$
p=-\sum_{m=1}^{\infty} \sum_{n=1}^{\infty} \frac{4 \gamma_{m n}}{\left(m^{2}+n^{2}\right) \pi^{2}} \phi_{m}(x) \phi_{n}(y),
$$

where

$$
\phi_{k}(x) \equiv \sin (k \pi x), \quad \gamma_{m n}=q_{m} q_{n}, \quad q_{n}=\frac{2}{n \pi}\left[1-(-1)^{n}\right] .
$$

From this solution, (3.10) indicates that, in this time range, the velocity components are clearly given by

$$
u=-\frac{p_{y}}{2}, \quad v=\frac{p_{x}}{2} .
$$


This solution provides the initial condition for the $E^{-\frac{1}{2}}$ time-scale solution.

Figure 8 shows a sample of contours for the starting-flow stream function, $p(x, y)$, defined in equation (3.15). Comparing these streamlines with the streak path image shown in figure 2(a), we see there is excellent qualitative agreement between theory and experiment at early times. A quantitative comparison is presented in figure 9, which shows the starting-flow velocity components $u(x, y)$ and $v(x, y)$, defined by equations (3.17) and (3.15), plotted with corresponding experimental data. To avoid saturation of data points and to allow the theoretical solution (indicated by the solid lines) to be clearly seen, measurements from four of the eight experiments are shown (see caption for details). Figure 9 (a) shows the velocity profile $u(y)$ along the central symmetry axis $x=1 / 2$, plotted against $y$. Similarly, figure $9(\mathrm{~b})$ shows $v(x)$ along the symmetry axis $y=1 / 2$, against $x$. Again, the agreement between experiment and theory is excellent. (Measurements from the four experiments not shown in figure 9 exhibit the same level of agreement with the theory.)

It is worth highlighting that the theoretical solution for starting flow presented in this section is an inviscid approximation based on free-slip conditions (but no penetration) at each of the container sidewalls. As a consequence, the theoretical solutions for $u$ and $v$, defined in (3.17), attain peak absolute values along the sidewalls (parallel to their direction). Of course, in reality, thin viscous shear layers develop along the sidewall boundaries through which the no-slip condition is satisfied. The effect of these shear layers is evident figure 4(a), by the thin layers of cyclonic vorticity that develop adjacent to the sidewalls, and in figure 9 by the rapid decay of the measured values of $u$ and $v$ near the sidewalls at, respectively, $x=0,1$ and $y=0,1$.

\subsection{Motion on the spin-up timescale for $t \sim \mathcal{O}\left(E^{-\frac{1}{2}}\right)$}

The equation of motion is as before (3.9), but on this long time scale, the first term in that equation is negligible, and integrating the second term leads to the equation of motion on this time scale,

$$
\nabla_{1}^{2} p+\frac{4}{S} p_{z z}=4
$$

However, the boundary conditions on the base and lid of the cylinder are altered from (3.11), since now Ekman suction needs to be accounted for. It is well known that the Ekman layers impose compatibility conditions on the outer velocity components, which are

$$
w= \pm \frac{1}{2} E^{\frac{1}{2}}\left(v_{x}-u_{y}\right) \quad \text { on } \quad z=\left\{\begin{array}{l}
0 \\
h .
\end{array}\right.
$$

Taking the time to be scaled as $t=E^{-\frac{1}{2}} \tau$, expanding all quantities as

$$
\mathbf{u}(x, y, z, \tau)=\mathbf{u}_{0}(x, y, z, \tau)+E^{\frac{1}{2}} \mathbf{u}_{1}(x, y, z, \tau)+\ldots,
$$

and substituting into (3.8) leads to

$$
u_{0}=-\frac{p_{0 y}}{2}, \quad v_{0}=\frac{p_{0 x}}{2}, \quad w_{0}=0, \quad \rho_{0}=-p_{0 z}, \quad w_{1}=-\frac{p_{0 z \tau}}{S} .
$$

Then, the leading-order Ekman conditions (3.19) become

$$
p_{0 z \tau}=\mp \frac{S}{4} \nabla_{1}^{2} p_{0} \quad \text { on } \quad z=\left\{\begin{array}{l}
0, \\
h,
\end{array}\right.
$$

which provide boundary conditions for (3.18).

The well-known result, reported in Walin (1969), that there is no boundary layer 
capable of transporting fluid vertically, means that there can be no penetration at the vertical boundaries, which then gives the additional sidewall boundary conditions

$$
p_{0}=0 \text { on } x=0,1,0 \leqslant y \leqslant 1 \text { and on } y=0,1,0 \leqslant x \leqslant 1 .
$$

A significant consequence of this boundary condition is that the tangential velocity component at the vertical walls is non-zero, which then means, according to (1.1), that the Ekman layers are not empty as they collide with those walls, so there is an eruption of fluid at the intersections of vertical and horizontal walls. Since as noted no vertical boundary layer can transport this erupting fluid, it must re-enter the interior by means of singular eruptions at those corners (Walin 1969). Hence, to account for this, (3.22) must be modified as

$$
p_{0 z \tau}=\mp \frac{S}{4} \nabla_{1}^{2} p_{0}+\mathcal{E} \quad \text { on } \quad z=\left\{\begin{array}{l}
0, \\
h,
\end{array}\right.
$$

where $\mathcal{E}$ denotes the singular eruptions. $\mathcal{E}$ must guarantee that there is no net vertical transport at either the bottom or top boundaries. Since $p_{0 z \tau}$ is the vertical velocity, $\mathcal{E}$ must be such that, at both $z=0, h$,

$$
\int_{0}^{1} \int_{0}^{1} p_{0 z \tau} \mathrm{d} x \mathrm{~d} y=0 .
$$

For convenience, we denote this requirement by $\overline{p_{0 z \tau}}=0$. So, $\mathcal{E}$ in (3.24) must be taken to assure that

$$
\overline{p_{0 z \tau}}=0 \quad \text { on } \quad z=\left\{\begin{array}{l}
0, \\
h .
\end{array}\right.
$$

Construction of a solution to (3.18) under conditions (3.23), (3.24) and (3.26) requires the following ansatz:

$$
\begin{gathered}
p_{0}(\mathbf{x}, \tau)=P(x, y, z, \tau)+z^{2} G(\tau)+z J(\tau)+K(x, y, \tau), \\
P(\mathbf{x}, \tau)=\sum_{m=1}^{\infty} \sum_{n=1}^{\infty} A_{m n}(z, \tau) \phi_{m}(x) \phi_{n}(y) \\
A_{m n}(z, \tau)=c_{m n}(\tau) \cosh \left[\mu_{m n}(z-h / 2)\right]-\frac{S \gamma_{m n}}{\mu_{m n}^{2}} \\
\nabla_{1}^{2} K=-\frac{8}{S} G \\
\mu_{m n}^{2} \equiv \frac{S}{4}\left(m^{2}+n^{2}\right) \pi^{2} .
\end{gathered}
$$

Substitution into the averaged boundary condition (3.26) gives

$$
\begin{gathered}
J_{\tau}+\left.\overline{P_{z \tau}}\right|_{z=0}=0, \\
J_{\tau}+2 h G_{\tau}+\left.\overline{P_{z \tau}}\right|_{z=h}=0 .
\end{gathered}
$$

Comparing with the early-time solution in $\S 3.4$, the initial condition for this set of equations is

$$
c_{m n}(0)=0 .
$$

Subtracting (3.28a) from (3.28b) and integrating with respect to $\tau$ gives

$$
G=-\frac{1}{4 h} \sum_{r=1}^{\infty} \sum_{s=1}^{\infty} \gamma_{r s} \mu_{r s} Q_{r s} c_{r s}
$$


since $G$ must also be zero at $\tau=0$. For convenience, we introduce

$$
P_{m n}=\cosh \left(\mu_{m n} h / 2\right), \quad Q_{m n}=\sinh \left(\mu_{m n} h / 2\right) .
$$

Integrating (3.28a) with respect to $\tau$ gives

$$
J=\frac{1}{4} \sum_{r=1}^{\infty} \sum_{s=1}^{\infty} \gamma_{r s} \mu_{r s} Q_{r s} c_{r s}=-G h .
$$

Then, we may solve $(3.27 d)$ for $K$, which gives

$$
K=2 G \sum_{m=1}^{\infty} \sum_{n=1}^{\infty} \frac{\gamma_{m n}}{\mu_{m n}^{2}} \phi_{m}(x) \phi_{n}(y) .
$$

Returning to (3.24), and using the orthogonality of the sine functions, we eventually obtain

$$
\begin{aligned}
\mu_{m n} Q_{m n} c_{m n \tau}+\mu_{m n}^{2} P_{m n} c_{m n}=\frac{1}{4} \gamma_{m n} \sum_{r=1}^{\infty} \sum_{s=1}^{\infty} \gamma_{r s} \mu_{r s} Q_{r s} c_{r s \tau} & \\
& +S \gamma_{m n}+\frac{\gamma_{m n}}{2 h} \sum_{r=1}^{\infty} \sum_{s=1}^{\infty} \gamma_{r s} \mu_{r s} Q_{r s} c_{r s}
\end{aligned}
$$

Note that, since $\mathcal{E}$ is singular in the corner, it has zero Fourier sine component, and so does not come into this equation.

We write the solution to (3.34) as

$$
c_{m n}(\tau)=\frac{\mathcal{C}_{m n}+C_{m n}(\tau)}{\mu_{m n} Q_{m n}},
$$

where $\mathcal{C}_{m n}$ is the particular solution, which is shown in Appendix A to be given by

$$
\mathcal{C}_{m n}=\frac{S \gamma_{m n}}{\lambda_{m n}(1-\sigma)}, \quad \sigma=\sum_{r=1}^{\infty} \sum_{s=1}^{\infty} \frac{\gamma_{r s}^{2}}{2 h \lambda_{r s}},
$$

where

$$
\lambda_{m n} \equiv \mu_{m n} \operatorname{coth}\left(\mu_{m n} h / 2\right) .
$$

The homogeneous solutions for $C_{m n}$ have time dependence $\exp (\Lambda \tau)$, and the eigenvalues shown in Appendix A to be solutions of

$$
\frac{\Lambda h+2}{4 h} \sum_{m=1}^{\infty} \sum_{n=1}^{\infty} \frac{\gamma_{m n}^{2}}{\Lambda+\lambda_{m n}}=1
$$

where it is also shown that all solutions are real and negative. In Appendix B, we indicate how these eigenvalues are computed. The first three eigenvalues for the experiments listed in table 1 are shown in table 2 .

The eigenfunction for a given $\Lambda_{\alpha}$, is given by

$$
C_{m n}^{(\alpha)}=\frac{\Lambda_{\alpha} h+2}{\Lambda_{\alpha}+\lambda_{m n}}
$$

The question of how these solutions may be combined to generate a solution that satisfies the initial condition is discussed in detail in Appendix B. 


$\begin{array}{ccccc}\text { Exp } & S & \Lambda_{1} & \Lambda_{2} & \Lambda_{3} \\ \mathrm{~A} & 26.4 & -19.2 & -33.2 & -36.5 \\ \mathrm{~B} & 8.40 & -10.6 & -18.7 & -20.5 \\ \mathrm{C} & 4.76 & -7.89 & -14.0 & -15.9 \\ \mathrm{D} & 2.34 & -5.42 & -9.78 & -10.7 \\ \mathrm{E} & 3.88 & -7.08 & -12.7 & -13.9 \\ \mathrm{~F} & 2.31 & -5.38 & -9.72 & -10.6 \\ \mathrm{G} & 4.84 & -7.96 & -14.1 & -15.5 \\ \mathrm{H} & 2.69 & -5.83 & -10.5 & -11.5\end{array}$

TABLE 2. The three smallest values of $\left|\Lambda_{\alpha}\right|$ for each of the eight experiments listed in table 1.

\subsection{Large- $\tau$ solution}

Since all members of $\left\{\Lambda_{\alpha}\right\}$ are real and negative, the particular solution corresponds to the large- $\tau$ limit, which can be shown from (3.36) to be

$$
\begin{array}{r}
\lim _{\tau \rightarrow \infty} p_{0}=\sum_{m=1}^{\infty} \sum_{n=1}^{\infty} \frac{\mathcal{C}_{m n}}{\mu_{m n} Q_{m n}}\left\{\cosh \left[\mu_{m n}(z-h / 2)\right]-\cosh \left(\mu_{m n} h / 2\right)\right\} \phi_{m}(x) \phi_{n}(y) \\
-\frac{1}{4} \sum_{r=1}^{\infty} \sum_{s=1}^{\infty} \gamma_{r s} \mathcal{C}_{r s}\left[\frac{z(h-z)}{h}\right]
\end{array}
$$

Inspection clearly shows that $p_{0}$, and hence $u_{0}=-p_{0 y} / 2, v_{0}=p_{0 x} / 2$ vanish at the top and bottom walls, and the central portion is the last to be spun up. In fact, this quantity evaluated at the center of the tank, $z=h / 2$, is, apart from a constant,

$$
\left.\lim _{\tau \rightarrow \infty} p_{0}\right|_{z=h / 2}=-\sum_{m=1}^{\infty} \sum_{n=1}^{\infty} \frac{S \gamma_{m n}}{\mu_{m n}^{2}}\left[\frac{1-\operatorname{sech}\left(\mu_{m n} h / 2\right)}{1-\sigma}\right] \phi_{m}(x) \phi_{n}(y) .
$$

From this result, we may obtain the vertical vorticity at $z=h / 2$, averaged over the square, as

$$
\left.\overline{\left(v_{x}-u_{y}\right)}\right|_{h / 2}=\left.\frac{1}{2} \overline{\nabla_{1}^{2} p_{0}}\right|_{h / 2}=\frac{2}{1-\sigma}-\frac{1}{2(1-\sigma)} \sum_{m=1}^{\infty} \sum_{n=1}^{\infty} \gamma_{m n}^{2} \operatorname{sech}\left(\mu_{m n} h / 2\right),
$$

which may easily be proved to be larger than 2 , the initial vorticity of the background rigid rotation. Thus, the vorticity at the center of the tank is actually larger than the initial vorticity-so the fluid there is less spun up than the initial, impulsive motion. To understand why, note that the leading-order vorticity equation for the interior motion is

$$
\left(v_{x}-u_{y}\right)_{t}=2 w_{z}
$$

with new vorticity created out of stretching. Integrating this equation from bottom to top,

$$
\frac{\partial}{\partial t} \int_{0}^{h}\left(v_{x}-u_{y}\right) \mathrm{d} z=2\left(\left.w\right|_{z=h}-\left.w\right|_{z=0}\right)
$$


Then integrating over the square $x-y$ domain, and recalling that the net vertical transport must be zero at each horizontal wall,

$$
\frac{\partial}{\partial t} \int_{0}^{h} \overline{\left(v_{x}-u_{y}\right)} \mathrm{d} z=0 \quad \Rightarrow \quad \mathcal{Z} \equiv \int_{0}^{h} \overline{\left(v_{x}-u_{y}\right)} \mathrm{d} z=2 h .
$$

As the regions near the horizontal walls become spun up, on the $E^{-\frac{1}{2}}$ time scale, $\overline{\left(v_{x}-u_{y}\right)}$ goes to zero there, and so to keep $\mathcal{Z}$ invariant in time, $\overline{\left(v_{x}-u_{y}\right)}$ must be larger than 2 at least somewhere in the tank. This invariance of $\mathcal{Z}$ seems to be a consequence of the Kelvin Theorem.

\subsection{Rayleigh layers on the sidewalls}

Unlike axisymmetric spin-up, where the tangential velocity and normal velocity at the outer wall are zero, and the non-zero vertical velocity must be taken to zero at the walls, here the tangential horizontal velocity component is non-zero at the vertical walls, and must be brought to zero there in a viscous layer. It turns out that, on this time scale, the side-wall layer is simply a Rayleigh layer. No viscous force/vortex-stretching distinguished limit can occur, since the vertical velocity is so small (see below) in this layer. To explore this boundary layer, on, say $x=0$, we write $x=\xi E^{\frac{1}{4}}$, and scale the velocity components and pressure as follows:

$$
u=E^{\frac{1}{4}} \hat{u}, \quad v=\hat{v}, \quad w=E^{\frac{3}{4}} \hat{w}, \quad p=E^{\frac{1}{4}} \hat{p} .
$$

Then, each hatted quantity is expanded in an asymptotic series

$$
\hat{\phi}=\hat{\phi}_{0}+E^{\frac{1}{4}} \hat{\phi}_{1}+E^{\frac{1}{2}} \hat{\phi}_{2}+\epsilon \hat{\phi}_{3}+\ldots, \quad \epsilon \ll E^{1 / 2} .
$$

Leaving out some details, it turns out on substitution into the full equations of motion that

$$
\begin{gathered}
\hat{u}_{0}=-\frac{\hat{p}_{0 y}}{2}, \quad \hat{v}_{0}=\frac{\hat{p}_{0 \xi}}{2}, \quad \hat{w}_{0}=-\frac{\hat{p}_{0 z \tau}}{S}, \\
\hat{u}_{1}=-\frac{\hat{p}_{1 y}}{2}+A(y, z, \tau), \quad \hat{v}_{1}=\frac{\hat{p}_{1 \xi}}{2}
\end{gathered}
$$

and $\hat{v}_{0}$ satisfies the differential equation

$$
\hat{v}_{0 \tau}+A_{y}=\hat{v}_{0 \xi \xi},
$$

which is clearly the equation for a Rayleigh layer. We note here that the nonlinear term is missing from this equation for $\epsilon \ll E^{\frac{1}{2}}$, since the asymptotic theory explored so far requires that restriction, (3.2). Examination of the outer solution shows that to match $A_{y}=-v_{0 \tau}(0, y, z, \tau)$, so the equation

$$
\hat{v}_{0 \tau}-v_{0 \tau}(0, y, z, \tau)=\hat{v}_{0 \xi \xi}
$$

may be solved by Laplace transforms,

$$
\hat{v}_{0}=\mathcal{L}^{-1}\left\{\mathcal{L}\left\{v_{0}(0, y, z, \tau)\right\}\left(1-e^{-\sqrt{s} \xi}\right)\right\} .
$$

It is apparent from table 2 that even at relatively small $S$, the last transient, due to $\Lambda_{1}$, has died away for $\tau \gtrsim \mathcal{O}(1)$, so that $C_{m n}$ is vanishingly small and only the "long-time" limit of the interior solution, $\mathcal{C}_{m n}$, remains numerically significant. In contrast, Rayleigh layers are still growing and developing for $\tau=\mathcal{O}(1)$. Hence, to facilitate comparison with experimental data, we adopt the following approach. Using (3.40) and (3.21) we see that 
the large- $\tau$ limit for $v_{0}$ is given by

$$
\begin{array}{r}
V_{0}(x, y, z)=\lim _{\tau \rightarrow \infty} v_{0}(x, y, z, \tau)=\sum_{m=1}^{\infty} \sum_{n=1}^{\infty} \frac{\mathcal{C}_{m n} m \pi}{2 \mu_{m n} Q_{m n}}\left\{\cosh \left[\mu_{m n}(z-h / 2)\right]\right. \\
\left.-\cosh \left(\mu_{m n} h / 2\right)\right\} \zeta_{m}(x) \phi_{n}(y),
\end{array}
$$

where

$$
\zeta_{m}(x)=\cos (\pi m x) .
$$

Hence, using the above argument, we have for $\tau=\mathcal{O}(1)$

$$
\hat{v}_{0} \approx V_{0}(0, y, z) \operatorname{erf}\left(\frac{\xi}{2 \sqrt{\tau}}\right) \text {. }
$$

For comparison with the experiments, using results from $\S 3.6$, we have the leading-order composite solution that includes the boundary layers on $x=0,1$ as

$$
\begin{array}{r}
v(x, y, z, \tau) \approx \sum_{m=1}^{\infty} \sum_{n=1}^{\infty} \frac{\mathcal{C}_{m n} m \pi}{2 \mu_{m n} Q_{m n}}\left\{\cosh \left[\mu_{m n}(z-h / 2)\right]-\cosh \left(\mu_{m n} h / 2\right)\right\} \zeta_{m}(x) \phi_{n}(y) \\
-V_{0}(0, y, z) \operatorname{erfc}\left(\frac{x}{2 \sqrt{E^{\frac{1}{2}} \tau}}\right)-V_{0}(1, y, z) \operatorname{erfc}\left(\frac{1-x}{2 \sqrt{E^{\frac{1}{2}} \tau}}\right)
\end{array}
$$

Notice that this solution is valid anywhere in the interior, except in the corner regions where there is a two-dimensional Rayleigh zone, whose solution is much more complicated than a simple error function. An expression similar to (3.50) may be written down for the velocity component in the $x$-direction, $u$, for which there are boundary layers on $y=0$ and $y=1$.

We now compare the theoretical, composite solution (3.50) with the velocity data obtained from the experiments. Note that, (3.50) accounts for the effects of Ekman suction and for the inward growth of the sidewall Rayleigh layers. It does not, however, account for the separation of the sidewall boundary layers, or the formation of, and flow within the corner-cell regions. Hence, a meaningful comparison of experimental data and theory along the (horizontal-plane) diagonal $q=\{(x, y): y=x$ for $x \in[0,1]\}$ is not possible. However, it is possible to make meaningful comparisons along the symmetry axes $x=1 / 2$ or $y=1 / 2$, where we expect the interior flow to be little affected by the corner cells. Here, we have chosen to show comparisons for the horizontal velocity profile $v(x)$ along the symmetry axis $y=1 / 2$, and at heights $z$ corresponding to the experimental data. So that results for a range of Burger numbers $(S)$ and heights $(z)$ can be shown, measurements of $v(x)$ taken from experiments A $(S=26.4, z=0.363 h)$, $\mathrm{D}(S=2.34, z=0.386 h), \mathrm{E}(S=3.88, z=0.588 h)$ and $\mathrm{H}(S=2.69, z=0.167 h)$ are plotted against $x$ in figures $10,11,12$ and 13 , respectively, and are compared with the corresponding theoretical velocity profiles (solid lines) generated using (3.50), again evaluated along $y=1 / 2$, and with values for $S$ and $z$ matched with the respective experiment parameters (see captions for details). As already noted, we expect (3.50) to be valid for $\tau=\mathcal{O}(1)$, and so the comparisons shown in each figure are for times between $\tau=1$ and 5 .

Figures 10, 11 and 12, correspond to heights about the cylinder's mid-depth. For these cases, the level of agreement between theory and experiment is generally excellent at each time shown. Moreover, a comparable level of agreement was also observed at intermediate times within this range, and for times up to $\tau \approx 8$. The comparisons at 
$\tau=1$ were generally not as good as those at later times, where there was a slight, but noticeable over-prediction of the measurements by the theoretical profile. A possible cause for this discrepancy could be that the effects of Ekman suction remain notable at this time, with the interior flow still approaching the large- $\tau$ limit defined by equation (3.40). However, as already noted, we believe this is unlikely given the large, negative values of the principal eigenvalues, $\Lambda_{1}$ (see table 2 ).

The comparisons shown in figure 13 correspond to the region close to the cylinder's base (at $z=0.167 h$ ). In this case, good agreement between theory and experiment was observed for times between $\tau \approx 1$ and $\tau \approx 2$ : Again, there was a slight but noticeable discrepancy evident at $\tau=1$, but excellent agreement for later times up to $\tau \approx 2$. This was not the case for times $\tau \gtrsim 2$, where the theory is seen to significantly over-predict the observed velocity data, as shown in figure $13(\mathrm{c})$. In $\S 2.2$ we showed that, for times $\tau \gtrsim 2$, the interior flow in the near-base region is notably affected by the reformation of cyclonic cells in each of the four corner regions, which are clearly evident in the streakline image shown in figure $3(\mathrm{e})$ (which corresponds to $\tau=2.2$ ). The result of the corner-cell reformation, which is not accounted for in (3.50), is that the fluid in the near-base region is spun-up more rapidly. In particular, note how the influence of the reformed corner cells is evident in the experimental data shown in figure 13(c) (which extracted along the symmetry axis $y=1 / 2$ ) by the change in sign of $v$ in the regions near the $x=0$ and $x=1$ sidewalls.

Finally, it is worth reiterating here that the theoretical results derived in $\S 3$ are based on the condition given in (3.2), i.e. that $\epsilon \ll E^{\frac{1}{2}} \ll 1$. However, the experiments reported here correspond to $E^{\frac{1}{2}} / \epsilon \approx 0.06$ to 0.31 , which clearly contradicts (3.2). Despite this, the theory and experimental data, in general, exhibit a remarkable level of correspondence.

\section{Sidewall boundary layers and eddy formation}

At this point, we have worked through the spin-up-time-scale dynamics, and the Rayleigh layers on the sidewalls. However, with a view to the parametric regime of this analysis, (3.2), we now investigate the time scale specified by $t=\epsilon^{-1} \bar{t}$. In a layer in which $x=(E / \epsilon)^{\frac{1}{2}} \bar{x}$, we make the following scalings:

$$
u=(E / \epsilon)^{\frac{1}{2}} \bar{u}, \quad v=\bar{v}, \quad w=(\epsilon E)^{\frac{1}{2}} \bar{w}, \quad p=(E / \epsilon)^{\frac{1}{2}} \bar{p}
$$

and then the equations with the leading-order terms under a typical boundary-layer approximation become

$$
\begin{gathered}
\bar{u}_{\bar{x}}+\bar{v}_{y}+(\epsilon E)^{\frac{1}{2}} \bar{w}_{z}=0 \\
\left(\epsilon^{3} / E\right)^{\frac{1}{2}}\left[v_{\bar{t}}+\bar{u} \bar{v}_{\bar{x}}+\bar{v} \bar{v}_{y}+(\epsilon E)^{\frac{1}{2}} \bar{w} \bar{v}_{z}\right]+2 \bar{u}+\bar{p}_{y}=\left(\epsilon^{3} / E\right)^{\frac{1}{2}} v_{\bar{x} \bar{x}} \\
-2 \bar{v}+\bar{p}_{\bar{x}}=\mathcal{O}\left((\epsilon E)^{\frac{1}{2}}\right), \\
\bar{p}_{z \bar{t}}=-S \bar{w} .
\end{gathered}
$$

Suppose for now that

$$
\epsilon=o\left(E^{\frac{1}{3}}\right),
$$

which includes the parametric restriction (3.2) in all of the foregoing theory. Writing the asymptotic expansion in this layer as

$$
\bar{v}=\bar{v}_{0}+\left(\epsilon^{3} / E\right)^{\frac{1}{2}} \bar{v}_{1}+\ldots,
$$



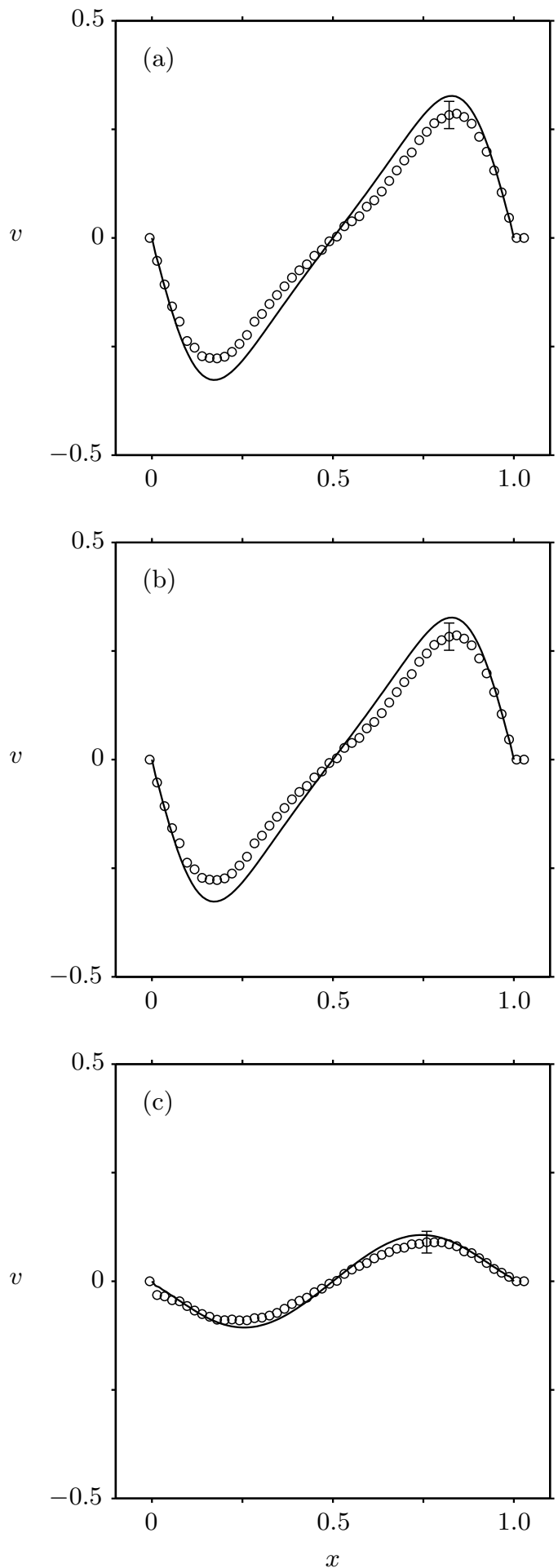

FiguRE 10. Experiment A $\left(\Omega=0.180 \mathrm{rad} / \mathrm{s}, \epsilon=0.107, S=26.4, z_{0}=0.363 H\right)$ : The solid lines show the composite solution $v(x, y, z, \tau)$, given by equation (3.50), evaluated along the symmetry axis $y=1 / 2$, at height $z=0.363 h$ and at dimensionless times (a) $\tau=1.0$, (b) $\tau=2.0$ and (c) $\tau=4.0$. Each curve is compared with corresponding experimental data (o). The errorbar shown in each plot is representative of the variability observed in the experimental data. 

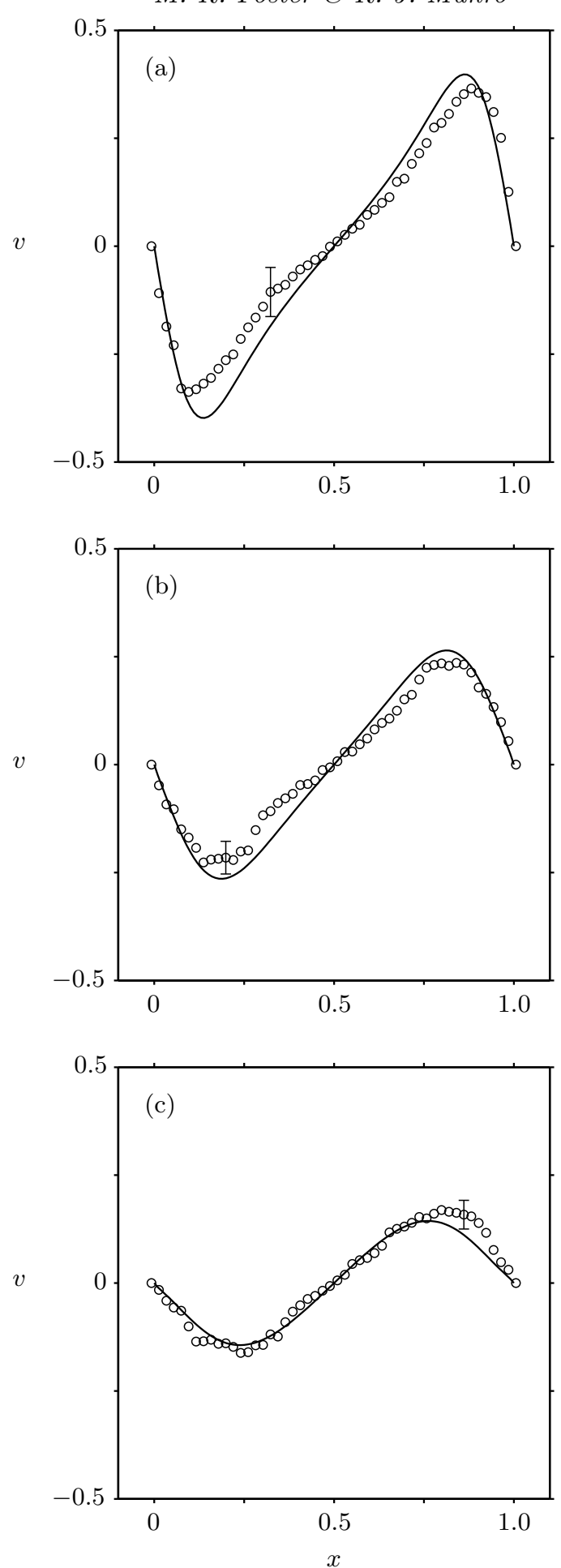

FiguRE 11. Experiment D $\left(\Omega=0.610 \mathrm{rad} / \mathrm{s}, \epsilon=0.0110, S=2.34, z_{0}=0.386 H\right)$ : The solid lines show the composite solution $v(x, y, z, \tau)$, given by equation (3.50), evaluated along the symmetry axis $y=1 / 2$, at height $z=0.588 h$ and at dimensionless times (a) $\tau=1.0$, (b) $\tau=2.5$ and (c) $\tau=5.0$. Each curve is compared with corresponding experimental data (o). The errorbar shown in each plot is representative of the variability observed in the experimental data. 

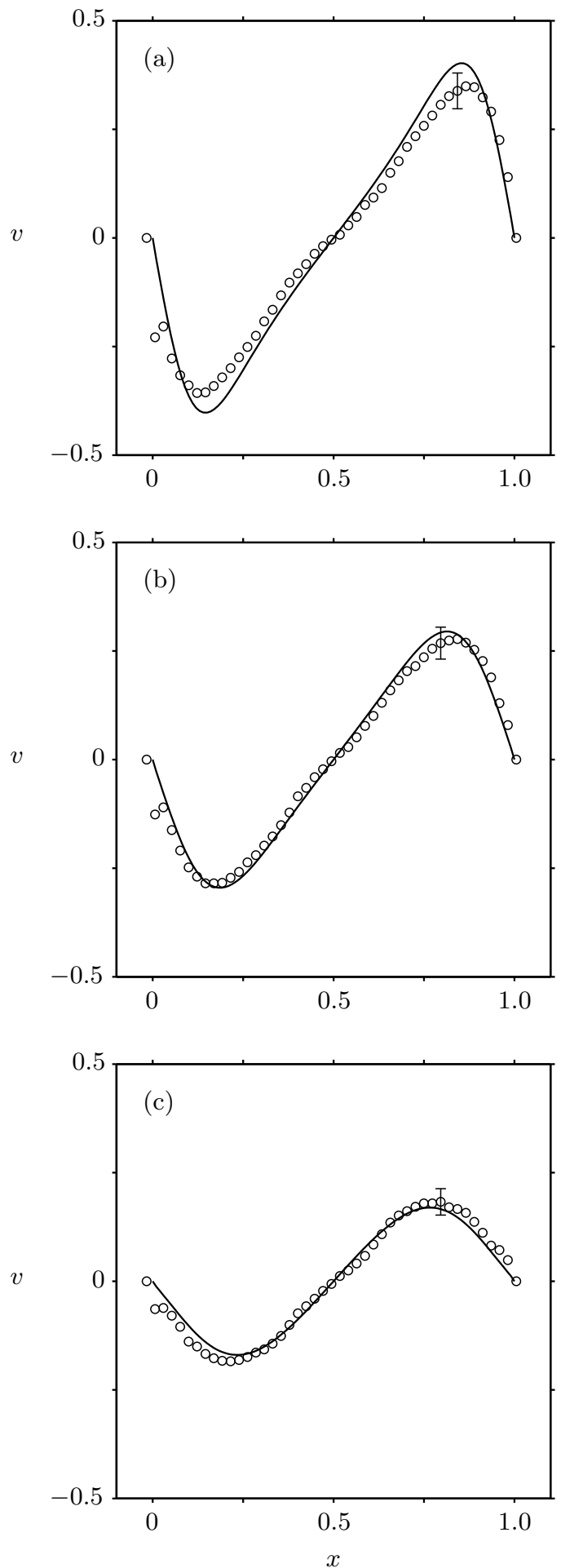

FiguRE 12. Experiment $\mathrm{E}\left(\Omega=0.405 \mathrm{rad} / \mathrm{s}, \epsilon=0.0203, S=3.88, z_{0}=0.588 H\right)$ : The solid lines show the composite solution $v(x, y, z, \tau)$, given by equation (3.50), evaluated along the symmetry axis $y=1 / 2$, at height $z=0.588 h$ and at dimensionless times (a) $\tau=1.0$, (b) $\tau=2.0$ and (c) $\tau=4.0$. Each curve is compared with corresponding experimental data (o). The errorbar shown in each plot is representative of the variability observed in the experimental data. 

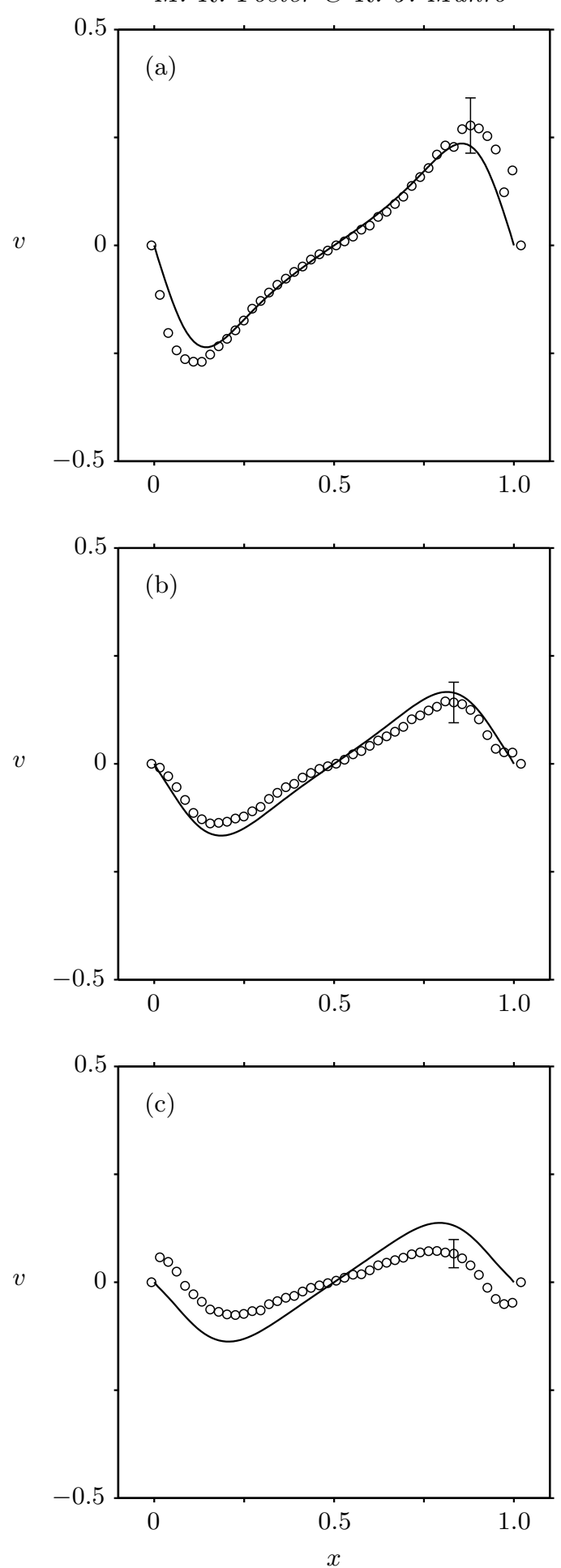

Figure 13. Experiment $\mathrm{H}\left(\Omega=0.494 \mathrm{rad} / \mathrm{s}, \epsilon=0.0101, S=2.69, z_{0}=0.167 \mathrm{H}\right)$ : The solid lines show the composite solution $v(x, y, z, \tau)$, given by equation (3.50), evaluated along the symmetry axis $y=1 / 2$, at height $z=0.588 h$ and at dimensionless times (a) $\tau=1.0$, (b) $\tau=2.0$ and (c) $\tau=2.6$. Each curve is compared with corresponding experimental data (o). The errorbar shown in each plot is representative of the variability observed in the experimental data. 
the leading-order equations simply reiterate $\bar{p}_{0}$ as a streamfunction, that is,

$$
\bar{v}_{0}=\frac{1}{2} \bar{p}_{0 \bar{x}}, \quad \bar{u}_{0}=-\frac{1}{2} \bar{p}_{0 y} .
$$

To next order, so long as $\epsilon \gg E$, we obtain first

$$
-2 \bar{v}_{1}+\bar{p}_{1 \bar{x}}=0 \text {. }
$$

Substituting into the continuity equation (4.2a) to this order, and integrating, we find that

$$
\bar{u}_{1}=-\frac{1}{2} p_{1 y}+A(y, z, \bar{t})
$$

The equation for $\bar{v}_{1}$ is

$$
\bar{v}_{0 \bar{t}}+\bar{u}_{0} \bar{v}_{0 \bar{x}}+\bar{v}_{0} \bar{v}_{0 y}+2 \bar{u}_{1}+\bar{p}_{1 y}=\bar{v}_{0 \bar{x} \bar{x}}
$$

Substituting from (4.7) into this equation leads to the Prandtl boundary-layer equation

$$
\bar{v}_{0 \bar{t}}+\bar{u}_{0} \bar{v}_{0 \bar{x}}+\bar{v}_{0} \bar{v}_{0 y}+A_{y}=\bar{v}_{0 \bar{x} \bar{x}}
$$

We see, on identifying $A_{y}$ with $-v_{0} v_{0 y}$ in the steady-state interior solution, this equation is precisely the Prandtl boundary-layer equation. Therefore, since the pressure gradient is adverse past $y=1 / 2$, the boundary layer will have a finite-time singularity on this time scale at some $y_{s}<1 / 2$, presumably causing the formation of the eddies that are seen in the experiments in the corners.

Notice that, if $\epsilon$ is of the same order as $E^{\frac{1}{3}}$, then the result is the same-the two terms in the series simply merge together. Suppose, however, that $\epsilon \gg E^{\frac{1}{3}}$ but still smaller than order one. In that case, the scalings may be re-done, the series recast, ending in precisely the same limit equation (4.9). So, it appears that the conventional Prandtl boundarylayer equation is the proper limit equation in this rotating frame for all small Rossby numbers. Finally, notice that if, as is the case in many of the experiments, $\epsilon / E^{\frac{1}{2}}$ is order one, then the spin-up time scale and the "eddy-formation time scale" of this section are identical, so there is in fact no Rayleigh layer on the spin-up time scale. That is to say, the sidewall boundary layer is fully nonlinear on the spin-up time scale. (Nonetheless, as noted elsewhere, using the composite expansion (3.50) which incorporates the Rayleigh layers gives good agreement with experiment!)

There is something important here that we believe has not been noted previously: In homogeneous spin-up, because of the vortex-stretching term (which is suppressed in our stratified case), it is easily confirmed that for Rossby number sufficiently small smaller than $\mathcal{O}\left(E^{\frac{1}{2}}\right)$ - there can be no such eddy formation. However, here, the Prantdl boundary-layer equation is always the proper boundary-layer equation regardless of the order of the Rossby number. That is, in the case of the problem considered here, eddies will always form at sufficiently long times, regardless of the order of $\epsilon$.

Finally, unlike axisymmetric spin-up, where the vertical-velocity layer is a standard "buoyancy layer", the non-zero $\bar{w}$ at the base of the Rayleigh layer can be shown to satisfy, on the spin-up time scale, the following equation:

$$
\bar{w}_{\hat{x} \hat{x} \tau}-S\left(\bar{w}-\bar{w}_{w}\right)=0, \quad \hat{x}=\frac{S^{\frac{1}{2}} x}{E^{\frac{3}{4}}},
$$

where $\bar{w}_{w}$ is the value of $\bar{w}$ from the Prandtl layer, evaluated at $\xi=0$. 


\section{Summary and final remarks}

In this paper, we have reported on a series of experiments in which linearly stratified fluid, initially rotating as a solid body within a closed square cylinder, is spun up to a new, slightly faster rotation rate. All experiments were conducted in the linear regime, where the Rossby number, $\epsilon=\Delta \Omega / \Omega$, was small. We have presented quantitative and qualitative comparisons of the experimental observations with an asymptotic linear theory. Both the theory and experiments focussed on the early time flow development, for times up to $\mathcal{O}\left(\Omega^{-1}\right)$, and on the subsequent flow development for times on the order of the spin-up time scale $T_{s}=E^{-\frac{1}{2}} \Omega^{-1}$, which for the experiments reported here was typically between 10-15 mins. Note, the flow development on the much longer diffusive time scale $T_{d}=E^{-1} \Omega^{-1}$, which here was typically $40 \mathrm{hrs}$, was not considered.

At early times, the flow adjustment is similar to that reported previously by van Heijst et al. (1990), for stratified spin-up from rest in a rectangular container, where it was shown that the initial flow is characterised by zero absolute vorticity. In the case considered here, where likewise the sidewall and Ekman boundary layers are still forming at early times, the relative flow is effectively inviscid, two-dimensional and conserves the vorticity of the initial condition, which for clockwise rotation is $\boldsymbol{\omega}^{*}=2 \Delta \Omega \hat{\mathbf{z}}$. Moreover, the relative flow is determined by the pressure field, which is a streamfunction with $u^{*}=-p_{y^{*}}^{*} / 2, v^{*}=p_{x^{*}}^{*} / 2$ and satisfies $\nabla^{* 2} p^{*}=4 \Delta \Omega$, with $p^{*}=0$ at the boundaries. The solution of this equation is straightforward and takes the form of a single anticyclonic cell, centred about the cylinder's axis, with closed streamlines that fill the flow domain. Comparisons between theory and experimental exhibit an excellent level of agreement on this time scale.

The next stage of the flow development is characterised by the formation and subsequent decay of weak columnar vortices in the cylinder's vertical corners, which were clearly evident in all of the experiments. It is shown that for times of order $T_{f}=\epsilon^{-1} \Omega^{-1}$, the horizontal-velocity boundary layers on the vertical sidewalls are described by Prandtl boundary-layer equations. Moreover, we conjecture that corner-cell formation is a result of a finite-time singularity in these Prandtl equations, developing in the region of adverse pressure upstream of corner regions. So, the cyclonic boundary-layer vorticity erupts from the sidewalls, resulting in the accumulation of separated fluid with cyclonic vorticity in each of the corner regions. Note that, in the experiments reported here $T_{f} / T_{s}=0.06$ to 0.31, and so the cyclonic corner cells are seen to form before Ekman suction has had a notable effect.

For times of order $T_{s}=E^{-\frac{1}{2}} \Omega^{-1}$ the growth of the corner cells has deformed the perimeter of the central anticyclonic cell, which is now circular in cross-section. Furthermore, the horizontal-velocity boundary layers on the vertical sidewalls are Rayleigh layers on this timescale. The subsequent spin-up of the central cell is due to the combined actions of Ekman suction through the base and lid boundary layers, and the inward growth of the sidewall Rayleigh layers. Furthermore, the eruption of spun-up, Ekman-layer fluid from the perimeter region of the cylinder's base and lid is crucial in the development of the spin-up dynamics on this time scale. An asymptotic result is formulated that accounts for each of these effects which is shown to exhibit excellent agreement with the experimental data, except very near the base (and lid) of the cylinder.

It is worth repeating here that the asymptotic theory is based on the condition that $0<\epsilon \ll E^{\frac{1}{2}} \ll 1$. In fact, in the experiments the time scale $T_{f}=\epsilon^{-1} \Omega^{-1}$ is significantly smaller than $T_{s}=E^{-\frac{1}{2}} \Omega^{-1}$ and so, as noted above, the corner cells form first on the spin-up time scale. Hence, the good experiment-theory agreement is very surprising. One difficulty is that conventional ordering arguments are very imprecise: Two terms in 
a given asymptotic series might have quite different "orders," but the term of smaller order could, for a given finite value of the small parameter, be numerically larger because the coefficient of the term of larger order turns out to numerically small. Since asymptotic series constructed in the fashion presented here are at this point in the development of the subject largely ad hoc, it is impossible to make any a priori estimate of a wider range of validity. Such methods have sometimes led to results that are valid in unexpectedly large regions of the parameter space. (See, for example, Jobe \& Burggraf (1974), whose "triple-deck" asymptotic correction to the Blasius formula for trailing-edge effects in flatplate drag agrees with experiment to Reynolds numbers of around a hundred, whereas the standard ordering arguments require a Reynolds number of several million.)

This work was supported by a Royal Society travel grant for MRF (grant number TG100245). The authors gratefully acknowledge Evgenia Korsukova for assisting with some of the experiments, and for the technical support provided by Damien Goy.

\section{Appendix A}

In $\S 3.5$, we have summarized the structure of the solution to the set of ordinary differential equations (3.34). Here, we articulate the details of those results.

\section{A.1. Particular solution to equation (3.34)}

Equation (3.34) obviously has a particular solution that obeys

$$
\lambda_{m n} \mathcal{C}_{m n}=\frac{\gamma_{m n}}{2 h} \sum_{r=1}^{\infty} \sum_{s=1}^{\infty} \gamma_{r s} \mathcal{C}_{r s}+S \gamma_{m n}
$$

where $\mathcal{C}_{m n}$ is defined in equation (3.35). Dividing by $\lambda_{m n}$ and multiplying by $\gamma_{m n}$, then summing over $m$ and $n$ determines the infinite sum in (A 1 ) to be given by

$$
\left[1-\sum_{r=1}^{\infty} \sum_{s=1}^{\infty} \frac{\gamma_{r s}^{2}}{2 h \lambda_{r s}}\right] \sum_{m=1}^{\infty} \sum_{n=1}^{\infty} \gamma_{m n} \mathcal{C}_{m n}=S \sum_{m=1}^{\infty} \sum_{n=1}^{\infty} \frac{\gamma_{m n}^{2}}{\lambda_{m n}} .
$$

Inserting this result into (A 1) and simplifying, we find that

$$
\mathcal{C}_{m n}=\frac{S \gamma_{m n}}{(1-\sigma) \lambda_{m n}}
$$

where

$$
\sigma \equiv \sum_{r=1}^{\infty} \sum_{s=1}^{\infty} \frac{\gamma_{r s}^{2}}{2 h \lambda_{r s}}
$$

\section{A.2. Homogeneous solution to equation (3.34)}

Since equation (3.34) has constant coefficients, we know that the homogeneous solutions have time dependence $\exp (\Lambda \tau)$, and substitution of that time dependence gives the nonlinear eigenvalue problem

$$
\left(\Lambda+\lambda_{m n}\right) C_{m n}=\frac{\gamma_{m n}}{4 h}(\Lambda h+2) \sum_{r=1}^{\infty} \sum_{s=1}^{\infty} \gamma_{r s} C_{r s} .
$$

Solving for $C_{m n}$, then multiplying by $\gamma_{m n}$ and summing over $m$ and $n$ leads to the eigenvalue equation (3.38),

$$
\frac{\Lambda h+2}{4 h} \sum_{m=1}^{\infty} \sum_{n=1}^{\infty} \frac{\gamma_{m n}^{2}}{\Lambda+\lambda_{m n}}=1
$$


Equation (A 5) may be rewritten as

$$
\frac{\Lambda+\lambda_{m n}}{\Lambda h+2} C_{m n}=\frac{\gamma_{m n}}{4 h} \sum_{r=1}^{\infty} \sum_{s=1}^{\infty} \gamma_{r s} C_{r s}
$$

Multiplying by $C_{m n}^{*}$ and summing over $m$ and $n$ leads to

$$
\sum_{m=1}^{\infty} \sum_{n=1}^{\infty} \frac{\Lambda+\lambda_{m n}}{\Lambda h+2}\left|C_{m n}\right|^{2}=\frac{1}{4 h} \sum_{m, n, r, s} \gamma_{m n} \gamma_{r s} C_{m n}^{*} C_{r s}
$$

where the $\sum$ notation on the right denotes a quadruple sum from 1 to $\infty$ over $m, n, r$, and $s$. Taking the complex conjugate of equation (A 7 ) by $C_{m n}$, then summing over $m$ and $n$ gives the equation

$$
\sum_{m=1}^{\infty} \sum_{n=1}^{\infty} \frac{\Lambda^{*}+\lambda_{m n}}{\Lambda^{*} h+2}\left|C_{m n}\right|^{2}=\frac{1}{4 h} \sum_{m, n, r, s} \gamma_{m n} \gamma_{r s} C_{m n} C_{r s}^{*}
$$

Interchanging $m$ with $r$ and $n$ with $s$ on the right side summations gives a result identical to the right side of (A 8). Subtracting this equation from (A 8) gives

$$
\left(\Lambda-\Lambda^{*}\right) \sum_{m=1}^{\infty} \sum_{n=1}^{\infty} \frac{h \lambda_{m n}-2}{|\Lambda h+2|^{2}}\left|C_{m n}\right|^{2}=0 .
$$

It may be easily shown that $h \lambda_{m n}-2>0$ for all values of $m$ and $n$, so the sum is positive and hence the only possibility is that $\Lambda$ is real.

Returning to equation (A 6), notice that the quantity $\lambda_{m n}>2 / h$, as follows from the properties of the hyperbolic cotangent. Take $\Lambda>0$, and then, $\Lambda+\lambda_{m n}>\Lambda+2 / h$. Therefore,

$$
(\Lambda+2 / h) \sum_{m=1}^{\infty} \sum_{n=1}^{\infty} \frac{\gamma_{m n}^{2}}{\Lambda+\lambda_{m n}}<\sum_{m=1}^{\infty} \sum_{n=1}^{\infty} \gamma_{m n}^{2},
$$

but this sum is equal to 4 from elementary considerations. Thus, we have

$$
\frac{1}{4}(\Lambda+2 / h) \sum_{m=1}^{\infty} \sum_{n=1}^{\infty} \frac{\gamma_{m n}^{2}}{\Lambda+\lambda_{m n}}<1
$$

However, (A 6) indicates that this quantity must be equal to one, giving a contradiction. Therefore, $\Lambda<0$. So all modes are damped, that is:

All members of $\left\{\Lambda_{\alpha}\right\}$ are real and negative.

\section{Appendix B}

There are two remaining questions related to the solution of the initial-value problem, detailed below.

\section{B.1. Numerical computation of $\left\{\Lambda_{\alpha}\right\}$}

We need to compute solutions to (A 6), which is done by series truncation. So, we replace ' $\infty$ ' in the double sum by $N$, which for convenience be now take to be odd only. Examination of this equation indicates that the polynomial obtained from (A 6) by terminating the sums at $N$ is $\mathcal{N}$, where $\mathcal{N}=(N+1)(N+3) / 8$, so for example, if $N=5$, there are 6 values for $\Lambda$. Typical results are shown in table 2 , where $N=151$ was used. As is typical with such series truncations, the modes with smallest $\left|\Lambda_{\alpha}\right|$ are insensitive to $N$. 
Changing to $N=201$ gives a change in $\Lambda_{1}$, for $S=1$, of $0.006 \%$. It is easy to confirm that $\mathcal{N}$ is even, and, from the computations, that $\Lambda_{1}$ and $\Lambda_{\mathcal{N}}$ are individual, but the $\mathcal{N}_{2}$ other eigenvalues are in pairs, both in the neighborhood of particular values of $\left(-\lambda_{m n}\right)$.

Once the eigenvalue has been determined, then the eigenvector is given, from (A 8), by

$$
C_{m n}=\text { constant } \times\left(\frac{\gamma_{m n}}{\Lambda_{\alpha}+\lambda_{m n}}\right) .
$$

Note that the constant is independent of $m$ and $n$.

\section{B.2. Initial-value problem solution}

So, the general homogeneous solution for $C_{m n}$ is then a sum over all eigenvalues, for a given $N$, and so is given by

$$
C_{m n}=\sum_{\alpha=1}^{\mathcal{N}} \frac{\chi_{\alpha} \gamma_{m n}}{\Lambda_{\alpha}+\lambda_{m n}} e^{\Lambda_{\alpha} \bar{t}}
$$

Then, noting the initial condition (3.29), we find that the requirement is

$$
\sum_{\alpha=1}^{\mathcal{N}} \frac{\chi_{\alpha} \gamma_{m n}}{\Lambda_{\alpha}+\lambda_{m n}}=-\mathcal{C}_{m n}=-\frac{S \gamma_{m n}}{\lambda_{m n}(1-\sigma)}
$$

which may be written as

$$
\gamma_{m n}\left[\sum_{\alpha=1}^{\mathcal{N}} \frac{\chi_{\alpha}}{\Lambda_{\alpha}+\lambda_{m n}}+\frac{S}{\lambda_{m n}(1-\sigma)}\right]=0 .
$$

Note that this equation is identically satisfied if either $m$ or $n$ is even. So, we have the following set of linear equations for the determination of $\left\{\chi_{\alpha}\right\}$,

$$
\sum_{\alpha=1}^{\mathcal{N}} \frac{\chi_{\alpha}}{\Lambda_{\alpha}+\lambda_{m n}}=-\frac{S}{\lambda_{m n}(1-\sigma)}, \quad \text { for odd } m, n .
$$

Examination of problem (B 5) shows that the series is slowly convergent, but because of the relatively large values of $\left|\Lambda_{\alpha}\right|$, good and instructive comparisons, shown in Figures 10, 11, 12 and 13, can be achieved without obtaining $\left\{\chi_{\alpha}\right\}$.

\section{REFERENCES}

Benton, E. R. \& Clark, A. 1974 Spin-up. Annu. Rev. Fluid Mech. 6, 257-280.

Duck, P. W. \& Foster, M. R. 2001 Spin-up of homogeneous and stratified fluids. Annu. Rev. Fluid Mech. 33, 231-263.

Greenspan, H. P. \& Howard, L. N. 1963 On a time-dependent motion of a rotating fluid. J. Fluid Mech. 17, 385-404.

Jobe, C. E. \& BurgGraf, O. R. 1974 The numerical solution of asymptotic equations of trailing-edge flow. Proc. Roy. Soc. A340, 91-111.

Munro, R. J., Foster, M. R. \& Davies, P. A. 2010 Instabilities in the spin-up of a rotating, stratified fluid. Phys. Fluids 22, 054108.

Oster, G. 1965 Density gradients. Scientific American 213(2), 70-76.

SAKURAI, T. 1969 Spin down problem of rotating stratified fluid in thermally insulated circular cylinders. J. Fluid Mech. 37(4), 689-699.

Spence, G. S. M., Foster, M. R. \& Davies, P. A. 1992 The transient response of a contained rotating stratified fluid to impulsive surface forcing. J. Fluid Mech. 243, 33-50.

Stewartson, K. 1957 On almost rigid rotations. J. Fluid Mech. 3, 17-26. 
van de Konijnenberg, J. A. \& van Heijst, G. J. F. 1997 Free-surface effects on spin-up in a rectangular tank. J. Fluid Mech. 334, 189-210.

van Heijst, G. J. F. 1989 Spin-up phenomena in non-axisymmetric containers. J. Fluid Mech. 206, 171-191.

van Heijst, G. J. F., Davies, P. A. \& Davis, R. G. 1990 Spin-up in a rectangular container. Phys. Fluids A 2, 150-159.

WALIN, G. 1969 Some aspects of time-dependent motion of a stratified rotating fluid. J. Fluid Mech. 36(2), 289-307. 